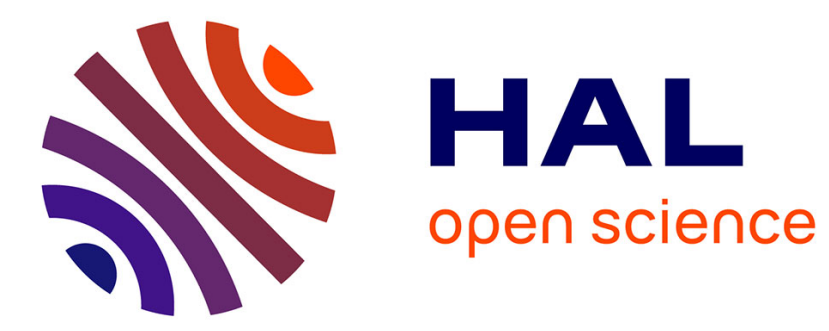

\title{
Scripting approach in hybrid organic-inorganic condensation simulation: the GPTMS proof-of-concept
}

\author{
Marek Maly, Paola Posocco, Maurizio Fermeglia, Sabrina Pricl
}

\section{To cite this version:}

Marek Maly, Paola Posocco, Maurizio Fermeglia, Sabrina Pricl. Scripting approach in hybrid organicinorganic condensation simulation: the GPTMS proof-of-concept. Molecular Simulation, 2008, 34 (10-15), pp.1215-1236. 10.1080/08927020802235706 . hal-00515044

\section{HAL Id: hal-00515044 \\ https://hal.science/hal-00515044}

Submitted on 4 Sep 2010

HAL is a multi-disciplinary open access archive for the deposit and dissemination of scientific research documents, whether they are published or not. The documents may come from teaching and research institutions in France or abroad, or from public or private research centers.
L'archive ouverte pluridisciplinaire HAL, est destinée au dépôt et à la diffusion de documents scientifiques de niveau recherche, publiés ou non, émanant des établissements d'enseignement et de recherche français ou étrangers, des laboratoires publics ou privés. 


\section{Molecular Simulation \\ Journal of \\ Experimental Nanoscience

\section{Scripting approach in hybrid organic-inorganic condensation simulation: the GPTMS proof-of-concept}

\begin{tabular}{|r|l|}
\hline Journal: & Molecular Simulation/Journal of Experimental Nanoscience \\
\hline Manuscript ID: & GMOS-2008-0058.R1 \\
\hline Journal: & Molecular Simulation \\
\hline Duthor: & 08-May-2008 \\
\hline Complete List of Authors: & $\begin{array}{l}\text { Maly, Marek; J. E. Purkinje University, Department of Physics; } \\
\text { Academy of Sciences of the Czech Republic, Institute of Chemical } \\
\text { Process Fundamentals } \\
\text { Posocco, Paola; University of Trieste, Department of Chemical } \\
\text { Engineering } \\
\text { Fermeglia, Maurizio; University of Trieste, Department of Chemical } \\
\text { Engineering } \\
\text { Pricl, Sabrina; University of Trieste, Department of Chemical } \\
\text { Engineering }\end{array}$ \\
\hline Keywords: & $\begin{array}{l}\text { GPTMS, condensation reaction, perl scripting, molecular dynamics, } \\
\text { thermophysical properties }\end{array}$ \\
\hline \hline
\end{tabular}

\section{SCHOLARONE Manuscripts}




\title{
Scripting Approach in Hybrid Organic-Inorganic Condensation Simulation: the GPTMS Proof-of- Concept
}

\author{
MAREK MALY $Y^{\mathrm{a}, \mathrm{b}}$, PAOLA POSOCCO ${ }^{\mathrm{c}}$, MAURIZIO FERMEGLIA $^{\mathrm{c}}$ AND SABRINA PRICL ${ }^{\mathrm{c}, *}$ \\ ${ }^{a}$ Department of Physics, Faculty of Science, J. E. Purkinje University, Usti nad Labem, Czech Republic; ${ }^{\mathrm{b}}$ Institute of \\ Chemical Process Fundamentals, Academy of Sciences of the Czech Republic, Prague, Czech Republic; ${ }^{\mathrm{c}}$ Department of \\ Chemical, Environmental and Raw Materials Engineering, University of Trieste, Trieste, Italy
}

\begin{abstract}
Silica-based hybrid organic-inorganic materials prepared by sol-gel chemistry exhibit unique chemical and physical properties. (3-glycidoxypropyl)trimethoxysilane (GPTMS)-based networks represent an archetype of this class of substances, with a vast range of applications. In the present study, a new computational recipe has been developed within Materials Studio software platform to generate atomistic models of GPTMS crosslinked networks. The methodology is based on molecular mechanics/dynamics schemes and assumes close proximity as a criterion for crosslinking reaction to occur. The COMPASS forcefield was selected for molecular model constructions, and two charge schemes - one obtained directly from the force field and one derived from quantum-chemical calculations - were employed and compared for the prediction of the final system thermophysical properties. Starting from fully-hydrolyzed GPTMS molecules, a realistic three-dimensional network was successfully constructed, including the presence of 4- and 6membered cyclic structures. Mechanical moduli and specific heat values estimated from equilibrated structures were selected as benchmarks for model/procedure validation. Overall, the simulation results are reasonable and in the range of experimental data available in the literature on similar systems. Thus, the proposed computational strategy has a good potential in the design and optimization of organic-inorganic hybrid materials.
\end{abstract}

Keywords: condensation reaction, GPTMS, molecular dynamics, Perl scripting, thermophysical properties

*Corresponding author. Email: Sabrina.Pricl@ dicamp.units.it

\section{Introduction}

Nanoscience and nanotechnology are opening new avenues in the fields of chemistry and physics of matter. In particular, the chance to create new, smart substances starting from a molecular level clearly constitutes an appealing way to design materials which possess targeted and well-defined macroscopic properties. In this scenario, the molecular building block - a nano-object - is usually an isolated entity (e.g., a molecule or an ensemble of nanoparticles) prepared at the nanometric scale which exhibits a specific chemical and/or 
physical property. Sol-gel formation technique constitutes one of the most actual, promising and convenient way to prepare nanomaterials, as it can be produced by using one-step processes which are flexible, efficient, and can be carried out in a wide range of operative conditions. Moreover, different devices characterized by different shapes - matrices, fibers, etc. - can be easily obtained.

Compared to other chemical elements, silicon is one of the most convenient and productive element for the preparation of the organically modified alkoxides required for the design and synthesis of hybrid organicinorganic (O/I) materials. A plethora of reasons speak in favor of the peculiar character of silicon, among which the transparency, thermal, and chemical stability of Si-O-Si networks, and the "sweet chemistry" involved in their synthesis $(1,2)$ are just a few. Generally speaking, the polycondensation reaction of alkoxysilanes results in a variety of structures, ranging from monodisperse silica particles to polymer networks, depending of the reaction conditions involved (3). In addition, copolymerization of alkoxysilanes of different functionalities makes it possible to tailor the ultimate material structure and performance. For example, tetrafunctional alkoxysilanes form densely crosslinked silica structure $\mathrm{SiO}_{2}$, trifunctional monomers polymerize to branched polysilsesquioxanes (PSSQOs) of the general formula $\mathrm{RSiO}_{3 / 2}$, whilst bifunctional alkoxysilanes generally yield linear polymer chains $\left(\mathrm{R}_{2} \mathrm{SiO}\right)_{\mathrm{n}}$. Ring formation is also a peculiar feature of alkoxysilanes polymerization reactions; clearly, the presence and amount of cyclic structures exert an influence on the ultimate structure and performances of the O/I hybrids (4-7).

Properties of the microheterogeneous organic-inorganic hybrids depend, to a good extent, on an interphase interaction determining the morphology. Strong interactions, for instance, leads to a reduction of the size of inorganic domains in the organic medium, and often improve the properties. Therefore, organofunctional trialkoxysilane monomers are used to prepare hybrid polymers and are employed as coupling agents, mainly in coating materials. 3-glycidyloxypropyltrimethoxysilane (GPTMS) is an organofunctional alkoxysilane monomer that can undergo both the sol-gel polymerization of the alkoxy groups and curing of the epoxy functionality to form a hybrid network with covalent bonds between organic and inorganic phases. Routinely, however, polymerization of GPTMS is carried out by a sol-gel process which leads to the formation of PSSQO structures with pendant, unreacted epoxy functionalities that are prone to later, eventual curing (4). O/I hybrid materials based on GPTMS have several important applications, including antiscratch coatings (8), contact lens materials (9), passivation layers for microelectronics (10), multifunctional coatings (11), and optical devices (12-14). GPTMS-based optical waveguides, in particular, are very promising materials because of the possibility to incorporate optically active organic molecules in a matrix that is dense at low temperature and with a high degree of microstructural homogeneity $(15,16)$. Accordingly, these extensive range of applications continue to attract various studies on GPTMS hybrid O/I systems. To obtain optimized formulations and efficient technological processes, however, extensive experimental campaigns must be carried out; further, some sound theories in conjunction to experiments must be developed, in order to gain some fundamental knowledge about the physical/chemical phenomena at the basis of the properties of these materials. On the other hand, on the spur of actual industrial competition, the number of lengthy and costly experiments must be drastically reduced, and the establishment of reliable, accurate theories is urgently needed, to be able to design molecular systems with fine-tuned, targeted properties.

Computer-based molecular simulations nowadays constitutes a versatile, efficient and reliable tool to achieve these goals. Indeed, these techniques can be of great help in reducing experimental hard work by sorting out useless trials and addressing the synthesis and characterization to more productive efforts. Accordingly, in this work we developed a computational strategy to obtain realistic molecular models of crosslinked polymer networks based on a molecular dynamics (MD) Perl script for the Materials Studio software platform. This methodology has been applied to mimick the formation of 3D hybrid O/I networks based on the condensation reaction of GPTMS under acid conditions. Further MD simulations were then carried out to predict some material properties (e.g., elastic constants and heat capacity) of the network structures thus obtained. 


\section{Computational details}

\subsection{Model building and general computational recipe}

Since the major aim of this work was to develop a script for simulating the generation of an hybrid O/I system based on GPTMS monomer, we decided to start by choosing the simplest conditions leading to the condensation network. Accordingly, we started by modeling a fully hydrolyzed GPTMS molecule, and mimic its condensation reaction under acid conditions (6), i.e., only the creation of Si-O-Si bonds between the available Si-O-H moieties takes place, leaving the epoxy groups unreacted (see Scheme 1).

For the generation of the final crosslinked system, the following general computational recipe was applied:

Step 1. The molecular model of the hydrolyzed GPTMS molecule was built, and its atoms were typed and charged using the COMPASS forcefield (ff) $(17,18)$. The molecular geometry was then optimized again using COMPASS ff. In order to test the eventual influence of the partial charge distribution on the physical properties of the final crosslinked system, we also assigned to the previously geometry optimized molecule a partial charge scheme obtained recharged using the quantum semiempirical method AM1-ESP as implemented in the Vamp toolbox of Materials Studio. At the end of Step 1, then, two molecular models of the hydrolyzed GPTMS monomer with the identical shape but different partial charges were obtained and used for further calculations.

Step 2. 100 hydrolyzed GPTMS molecules were packed into a simulation box under periodic boundary conditions using the Amorphous Cell builder modulus of Materials Studio. The initial density of the liquid mixture was set to $1.57 \mathrm{gcm}^{-3}$, a value estimated from the average experimental density of the final crosslinked system assuming an ideal case of $100 \%$ condensation $\left(1.35 \mathrm{gcm}^{-3}, 19\right)$. To start the condensation reaction/networking process from a representative initial system, 100 different simulation boxes were independently created for each charge scheme considered (i.e., 200 3D cells were obtained overall). After geometry relaxation of each 3D box, the one with the lowest energy value was selected, - namely structures I one for each charge scheme, for running the molecular dynamics (MD) simulations. Finally, in order to determine the eventual influence of the choice of the initial structure on the simulation results, two further, independent MD simulations for each charging case on structures characterized by approximately the same energy value of structure I after relaxation - namely structures II, were also conducted and analyzed.

Step 3. The selected 3D boxes containing the initial GPTMS monomer systems were first subjected to a geometry optimization (2000 steps); then simulated annealing procedure was applied (20-25) (8 cycles of $1000 \mathrm{MD}$ steps , temperature range $200 \mathrm{~K}-500 \mathrm{~K}$ ). 5000 steps of molecular dynamics a room temperature $(298 \mathrm{~K})$ were carried out. All simulations were conducted in the canonical (NVT) ensemble. After preliminary trials (results not shown), an integration time step of 0.2 fs finally was selected, giving a total annealing time of $1.6 \mathrm{ps}$, and an overall MD time of $1 \mathrm{ps}$.

Step 4. The distances between the reactive atoms $(\mathrm{O}$ and $\mathrm{H}$ atoms from each $\mathrm{OH}$ group linked to the $\mathrm{Si}$ atom, see Scheme 1) were measured and ordered in an increasing order. The three closest pairs of reactive atoms whose distances were smaller than the selected reactive cutoff distance $(3 \AA)$ were identified, and between the corresponding $\mathrm{Si}$ atoms new $\mathrm{Si}-\mathrm{O}-\mathrm{Si}$ bonds were created. An equivalent number of water molecules were deleted from the system (see Figure 1). The reactive cutoff distance was increased during the networking formation from $3 \AA$ to $6 \AA$. Since the presence of small, strained rings (i.e., with less than $4 \mathrm{Si}$ atoms) in the final system is not found experimentally in hybrid O/I systems based on GPTMS $(4,26)$, the necessary restrictions were implemented in the corresponding script, as described in details in the following paragraph.

Step 5. Steps 3 (referred to the actual cell) and 4 were repeated until no more pairs of reactive atoms satisfying all criteria were detected in the system. 


\subsection{Condensation reaction script}

The whole network formation procedure was achieved by writing a Perl script using the object library available in Materials Studio v. 4.1. In what follows, for the sake of clarity and brevity, we will limit the description to the most important technical parts of the final version of the script. Further details are available upon request. Each condensation simulation starts by loading the initial structure obtained at Step 2 and its association to a proper variable to be used for accessing all objects present in the structure itself. The corresponding command is:

my $\$ d o c=\$ D o c u m e n t s\{" n a m e . x s d "\} ;$

The next action consists in deleting all existing sets in the initial structure, i.e.:

my \$sets = \$doc->UnitCell->Sets;

foreach my \$set (@\$sets) \{

\$set->Delete;

\}

This is an important issue as, in some of the next steps, some new sets will be created with respect to predefined reactive atoms; these sets, in turn, will be used to create the relevant close contacts and, ultimately, bonds. Accordingly, the presence of other sets has to be avoided.

Since the simulations are performed under 3D periodic conditions, one of the filters DisplayRange, UnitCell or AsymmetricUnit should be applied to access any object in the document. These filters define finite sets of accessible objects in the document. For example, the DisplayRange filter allows to access the items which are displayed when the document is viewed in the Materials Studio Visualizer, whilst the UnitCell filter returns objects which are unique with respect to periodic translation of the lattice. Having tested both the UnitCell and DisplayRange filters, we selected to use the former. From preliminary tests performed on the considered systems (data not shown) it follows that both filters can be employed for scripting. The results are very similar in terms of physical properties of the final structures, and a criterion for the alternative choice of these filters will be the subject of a more detailed study.

It is useful to create a simple variable which will maintain all the atoms instead using

\section{\$doc->UnitCell->Atoms;}

This association is done by this command:

my \$atoms = \$doc->UnitCell->Atoms;

Another fundamental step that has to be undertaken is proper labeling of all the important chemical entities which, for the specific system considered here, are the linked Si-O-H atoms. Accordingly, all Silicon atoms were labeled $\mathbf{S i}$, the oxygen atoms covalently bonded to it $\mathbf{O}$, and the reactive hydrogens $\mathbf{H}$. The whole Perl code for this action is listed below:

foreach my \$atom (@\$atoms)\{

if(\$atom->ElementSymbol eq "Si")\{

\$atom->Name = "Si";

foreach my \$atom1 (@\{\$atom->AttachedAtoms\})\{ 
if( \$atom1->ElementSymbol eq "O" \&\& \$atom1->Name ne "RO")\{\$atom1->Name = "O"; foreach my \$atom2 (@\{\$atom1->AttachedAtoms\})\{ if( \$atom2->ElementSymbol eq "H")\{\$atom2->Name = "H"; \}

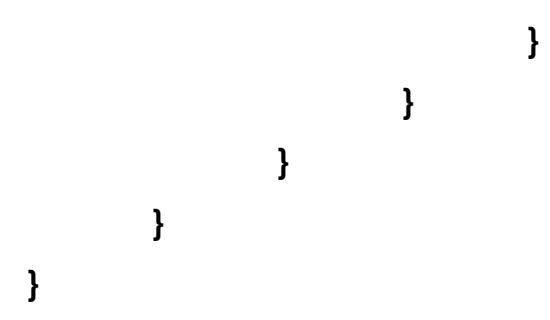

The RO label was chosen for the oxygen atoms bridging two $\mathrm{Si}$ atoms (i.e., Si-O-Si). Since it may be necessary to restart the whole script several times, it is essential to avoid relabeling RO oxygens to $\mathbf{0}$, and this justifies the choice of the label RO in the above piece of code.

Before performing any calculation, all relevant parameters for Geometry Optimization (GO), Annealing (AN) and Molecular Dynamics (MD) must be set, and each proper setting saved to a file (*.xms). This setting file must be placed later in the same directory where the Perl script is located. Then, the command for loading the parameter file:

\section{Modules->Forcite->LoadSettings("name");}

must be included in the script. This avoids the tedious repeating of setup operations. As can be seen from the command above, the Forcite module of Materials Studio was employed in the calculations performed in this work, as scripting cannot be implemented in the Discover engine available in version 4.1 of Materials Studio. Direct commands inside the script are then used to set some important calculation parameters for running the calculations. The settings and running commands for GO, AN, and MD) calculations employed in the present study are reported below:

Modules->Forcite->ChangeSettings(["ChargeAssignment" => "Use current"]);

Modules->Forcite->Calculation->Run(\$doc, Settings(Task => "Geometry Optimization", Maxlterations => 2000));

Modules->Forcite->Calculation->Run(\$doc, Settings(Task => "Anneal",TimeStep => 0.2, AnnealCycles => 8, EnergyDeviation $=>2000000)$ );

Modules->Forcite->Calculation->Run(\$doc, Settings(Task => "Dynamics",TimeStep => 0.2, Ensemble3D => "NVT", NumberOfSteps => 5000, EnergyDeviation => 2000000$)$ );

When a trajectory file (*.xtd) is created as output of the AN and MD simulations, the \$doc variable is automatically reinitialized, and from that moment it represents the whole trajectory of the system. Since just the last frame from each of these trajectories is needed as input for subsequent calculations, this last fame is used for reinitializing the document variable \$doc after each AN/MD run. Furthermore, in the case of MD runs (after which Step 4 is applied, see above), another important reason to update \$doc variable to the last frame of the trajectory can be envisaged. Since some atoms are to be deleted from the system during the condensation reaction leading to network formation, (i.e., $\mathrm{H}_{2} \mathrm{O}$ molecules, see Figure 1), the atomistic file (*.xsd) and not the trajectory file (*.xtd) has to be used as, once the number of atoms in actual frame is altered, the entire trajectory is invalidated. The complete sequence of commands in AN, MD part of our script is as follows:

Modules->Forcite->Calculation->Run( $\$$ doc, Settings (Task $=>$ "Anneal",TimeStep $=>0.2$, AnnealCycles $=>8$, EnergyDeviation $=>2000000)$ ); 
my $\$$ numFrames = \$doc->Trajectory->NumFrames;

\$doc->Trajectory->CurrentFrame = \$numFrames;

my \$LastFrame = Documents->New("After-ANN.xsd");

\$doc -> Discard;

\$LastFrame -> CopyFrom(\$doc);

$\$ d o c=\$$ LastFrame;

Modules->Forcite->Calculation->Run(\$doc, Settings(Task => "Dynamics",TimeStep => 0.2, Ensemble3D => "NVT", NumberOfSteps $=>5000$, EnergyDeviation $=>2000000)$ );

my $\$$ numFrames = \$doc->Trajectory->NumFrames;

\$doc->Trajectory->CurrentFrame = \$numFrames;

my \$LastFrame = Documents->New("After-MD.xsd");

\$LastFrame -> CopyFrom(\$doc);

$\$$ doc $->$ Discard;

$\$$ doc $=\$$ LastFrame;

On the first line of the \$doc updating code, the new variable \$numFrames is created, and the number of frames of the considered trajectory is assigned to it. Then, the last frame of the trajectory is set as the actual one. Afterwards, the empty atomistic file is created (After-ANN.xsd or After-MD.xsd in the above routine) and associated to the variable \$LastFrame. In the next line of the code, the last frame of the actual trajectory is copied into the new atomistic file; subsequently, the current trajectory variable is discarded, clearing this document (i.e., the trajectory file) from the computer memory. This obviously does not imply that the trajectory file which is represented by this variable is deleted. Each trajectory file, together with the corresponding (After-ANN.xsd or After-MD.xsd) files, are saved in the corresponding Materials Studio project. The last step is updating the document variable \$doc by variable \$LastFrame, where the last frame from the previous trajectory is stored.

Since Steps $\mathbf{3}$ and $\mathbf{4}$ are repeated several time during the entire simulation recipe, the After-ANN.xsd and AfterMD.xsd files are automatically numbered (e.g., After-ANN (2), After-ANN (3) etc.) by Materials Studio. The same automatic procedure is applied to the corresponding trajectories files. No saving instructions then need to be implemented since Materials Studio includes all created files automatically into the open project.

Now let us describe a little more in details Step 4, which includes i) the analysis of the distances of the pairs of reactive atoms, ii) the creation of new Si-O-Si bonds, and iii) the deletion of $\mathrm{H}_{2} \mathrm{O}$ molecules from the system. In order to make the system react, the optimal close contacts between the atoms involved in the condensation reaction have to be realized; accordingly, the atom pairs between which close contacts should take place have to be defined. Given the chemistry of the systems considered in this work, where condensation takes place between $\mathrm{Si}-\mathrm{O}-\mathrm{H}^{\cdots} \mathrm{H}-\mathrm{O}-\mathrm{Si}$ groups (see Scheme 1), only $\mathrm{O}-\mathrm{H}$ close contacts are needed, where $\mathrm{O}$ is the name for the oxygens bound to the $\mathrm{Si}$ atoms, and $\mathrm{H}$ designs the hydrogens linked to the $\mathrm{O}$ oxygens. The part of our script that creates these close contacts is written below:

\section{\# Create arrays to store the reactive atoms in}

my @reactive_0;

my @reactive_H;

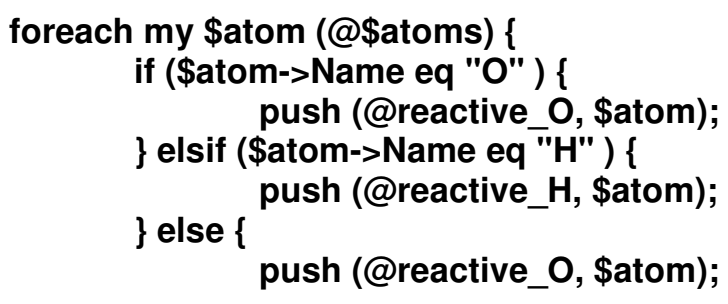


The above piece of code creates two atom arrays, reactive_O and reactive_H, which, intuitively, store all atoms except atoms named $\mathbf{H}$ and named $\mathbf{0}$, respectively. Since close contact restrictions can be applied only to sets, however, it is necessary to create relevant sets based on previously created atomic arrays. This can be done by the following commands:

my \$reactive_O_set=\$doc->CreateSet("reactive_0",|@reactive_0); my \$reactive_H_set=\$doc->CreateSet("reactive_H",|@reactive_H);

Then, the ExclusionMode is set to 'Set' value, which should guarantee that creation of close contacts between atoms inside each defined set is forbidden. Clearly in this study only the close contacts between atoms named $\mathbf{O}$ and $\mathbf{H}$ are expected, since these atoms are in different sets. The command that make the above described exclusion mode setting is the following:

\section{Tools->BondCalculation->ChangeSettings([ExclusionMode => 'Set']);}

Unfortunately, this elegant trick works perfectly only under non-periodic conditions (e.g., a non-periodic superstructure). In the case of a system under periodic boundary conditions, such as in the present work, it is necessary to introduce some further filtering to eliminate all the non $\mathbf{O}-\mathbf{H}$ close contacts (see the code part for the creation of GoodCloseContacts array listed below). Nevertheless, given the 3D symmetry characteristics of our system, this approach can be successfully applied, ultimately resulting in a substantial reduction computer memory.

The last step necessary for calculating close contacts is the setting of DistanceCriterionMode, MinAbsoluteDistance and MaxAbsoluteDistance parameters. In our case, the following setting was employed:

Tools->BondCalculation->ChangeSettings(Settings( DistanceCriterionMode =>"Absolute", MinAbsoluteDistance $=>0.0$, MaxAbsoluteDistance $=>3$ ));

At this point, predefined close contacts can be calculated according to:

\section{\$doc->CalculateCloseContacts;}

Again, it is appropriate to create a variable(s) which will represent the set of created close contacts: \$CloseContacts:

my $\$$ CloseContacts $=\$$ doc->UnitCell ->CloseContacts;

For the next procedure, mainly in order to save significant time and memory in loop operations, the creation and use of an arrays, such as GoodCloseContacts, in which all the necessary information can be stored, needs to be created:

\section{my @GoodCloseContacts;}

The code for the initialization of the GoodCloseContacts array is listed below:

foreach my \$CloseContact (@\$CloseContacts) \{

my \$Name1= \$CloseContact- $>$ Atom1->Name;

my $\$$ Name2 = \$CloseContact->Atom2->Name; 
my \$Atom1 = \$CloseContact->Atom1;

my \$Atom2 = \$CloseContact->Atom2;

if ( (\$Name1 eq "O") \&\& (\$Name2 eq "H") || (\$Name1 eq "H") \&\& (\$Name2 eq "O") ) \{

push(@GoodCloseContacts,[1,\$Name1,\$Name2,\$Atom1->X,\$Atom1->Y,\$Atom1->Z,\$Atom2->X,

\$Atom2->Y,\$Atom2->Z,\$CloseContact->Length,\$CloseContact->Atom1,\$CloseContact->Atom2]);

\}

In the above code, each element of the GoodCloseContacts array has the structure [status,Name1,Name2, x1,y1,z1,x2,y2,z2,Length,Atom1,Atom2], where status represents a variable which denotes if a given close contact can take place or not at a given instant. Name1 and Name2 are the names of the atoms which belong to that given close contact. $\mathbf{x} \mathbf{1}, \mathbf{y} \mathbf{1}, \mathbf{z 1}, \mathbf{x 2}, \mathbf{y} \mathbf{2}, \mathbf{z 2}$ are the coordinates of these atoms, Length denotes the length of the given close contact, and finally Atom 1 and Atom 2 are the atom objects which belongs to the close contact. The names of these atoms are stored in the array as separate variables (i.e., Name1 and Name2); however, they are accessible through the atom objects. This last action was taken just to save some time, since atom names will be frequently used in the analysis section (see below).

It is also worthwhile to note that the filtering of non $\mathbf{O}-\mathbf{H}$ close contacts discussed previously is included in the piece of code listed above; accordingly, the GoodCloseContacts array contains only $\mathbf{O}-\mathbf{H}$ close contacts. However, in order to simulate a network-forming condensation reaction, only those $\mathbf{O}-\mathbf{H}$ close contacts which are created between two different GPTMS molecules must be taken into account. Therefore, it is necessary to filter out the $\mathbf{O}-\mathbf{H}$ close contacts which are created between $\mathrm{O}$ and $\mathrm{H}$ atoms belonging to the same GPTMS molecule. This can be simply achieved by analyzing the array GoodCloseContacts and checking whether the species Atom1 and Atom2 belong to the same GPTMS molecule or not. In the affirmative, the variable status must be changed from 1 to -1 , and all the subsequent analysis must be carried out only on those close contacts which have variable status equal to 1 .

The way to identify the molecular object to which a given atom belongs is using:

\section{\$atom1->Ancestors->Molecule}

Unfortunately, at present, there is no direct method to obtain the index of any given molecule in a molecular model. Thus, a way to circumvent the problem is to identify two different molecules by their different center of mass. To access the coordinates of the center of mass of any given molecule, the following construction can be used:

my $\$ \mathrm{x} 1=$ \$atom1->Ancestors->Molecule->Center->X;

my \$y1 = \$atom1->Ancestors->Molecule->Center->Y;

my \$z1 = \$atom1->Ancestors->Molecule->Center->Z;

Finally, the GoodCloseContacts is ordered by increasing close contact length which, in each element of this array, is represented by the Length variable.

At this point, the new Si-O-Si bonds building procedure can begin. As the whole code for this section is big, and the detailed description of each part will exceed the scope of this article, it will only be summarized and briefly commented below.

The first close contact (by index) in the array GoodCloseContacts which has the status variable equal to 1 (i.e., the shortest one, see above) is chosen. Two new variables $\mathbf{\$ O}$ and $\mathbf{\$ H}$ are created, which are initialized by the atoms Atom1 and Atom2 from the selected GoodCloseContacts element. All the remaining atoms involved in the bond formation are then identified, and assigned to the proper variables $(\mathbf{\$ H} 1, \mathbf{0 1}, \mathbf{\$ S i}$, and $\mathbf{\$ S i 1}$, respectively). Figure 2 illustrates the association of these variables to the corresponding atoms. 
According to the bonding scheme of Figure 2, at the beginning only the two atoms which belongs to the chosen close contact ( $\$ \mathbf{O}$ and $\mathbf{\$ H}$, respectively) are considered. Next, the hydrogen atom $\mathbf{\$ H} \mathbf{1}$, which will be deleted together with atoms $\$ \mathrm{O}$ and $\$ \mathrm{H}$, from the system due to the formation of a $\mathrm{H}_{2} \mathrm{O}$ molecule, must be identified (see Figure 2). It is important to note that, in the real process, the water formed during the condensation reaction remains in the reaction environment, and eventually contributes to the hydrolysis of nonhydrolyzed GPTMS molecules. In the system considered here, however, the GPTMS monomers were fully hydrolyzed (a realistic condition which can be easily achieved experimentally). Therefore, water plays no additional role in the process, and does not need to be further considered. Next, atoms $\$ \mathbf{O} 1, \$ \mathrm{Si}$, and $\$ \mathrm{Si1}$ must be identified, in order to create the new bond $\$$ Si-\$O1 (see Figure 2). This identification process can be carried out resorting to the AttachedAtoms function. Below, the piece of code for the identification of the \$H1 and $\mathbf{\$ S i}$ atoms is listed, as an example:

my $\$ \mathrm{Si} ;$

my \$H1;

foreach my \$at $(@\{\$ 0->$ AttachedAtoms $\})\{$

if(\$at->Name eq "Si")\{\$Si=\$at; $\}$ else $\{\$ \mathrm{H} 1=\$ a t ;\}$

\section{\}}

As mention in the introduction, although the structure of a GPTMS-based hybrid O/I network obtained under acid catalysis is similar to that of a crosslinked polymer network, some cyclic structures can be present $(4,26)$. In order to apply some restrictions related to ring size in the formation of eventual cyclic structures, a check whether the bonding between the $\$ \mathbf{S i}$ and $\$ \mathbf{O} 1$ atoms will not result in the closure of a strained ring (i.e., with less than $4 \mathrm{Si}$ atoms) must be performed. This leads to the necessity of identifying atom $\$$ Si1 (see Figure 2); this operation could also serve graphical purposes: for example, the need of changing graphical style for both bonds $\$$ Si-\$O1 and \$01-\$Si1 in order to differentiate them, for example, from other bonds.

Should every condition described above be satisfied, the new bond $\$ \mathbf{S i}$ - \$O1 is created at this stage with the command:

\section{\$doc->CreateBond(\$Si, \$O1, "Single");}

otherwise, the next available close contact in the GoodCloseContacts array is selected, and the whole procedure outlined above is repeated.

As said, once the new Si-O bond is created, a water molecule is released. In our model, this corresponds to deleting atoms $\$ \mathbf{O}, \mathbf{\$ H}$, and $\mathbf{\$ H} \mathbf{1}$ from the system. To accomplish this goal, instead of deleting the required atoms directly (e.g., using commands such as \$O->Delete, etc., which can generate problems, especially when using periodic boundary conditions, as once some individual atoms are deleted, all its periodic images are also deleted from the set of close contact atoms).

push (@AtomsToDelete,\$H); push (@AtomsToDelete, $\$ 0)$; push (@AtomsToDelete,\$H1);

After the end of the \$i loop, a set based on the AtomtoDelete array is created, and all the atoms which are deleted from the system according to the reaction scheme will be cancelled at once and in a safe way, according to:

my \$AtomsToDelete_Set=\$doc->CreateSet("AtomsToDelete",|@AtomsToDelete); \$AtomsToDelete_Set->Items->Delete; 


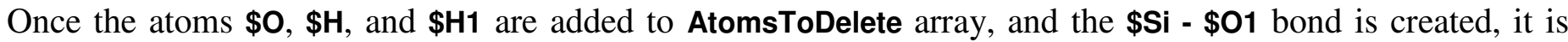
appropriate to analyze the whole GoodCloseContacts array from the actual index $\$ \mathrm{i}$ to the end, and switch the variable status to -1 in all those cases in which some of the atoms $\mathbf{\$ O}, \mathbf{\$ O} \mathbf{\$} \mathbf{\$ H}$, and $\mathbf{\$ H} 1$ are periodically equivalent to Atom1 or Atom2.

For checking periodic equivalence of two atom, the following function was created:

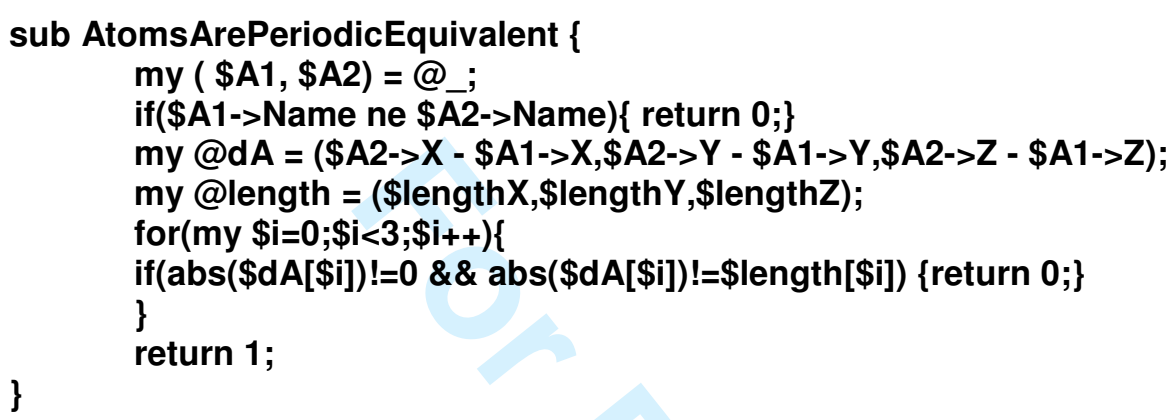

\#The variables \$lengthX, \$lengthY and \$lengthZ denotes the length of the sides of the periodic box.

Figure 3 shows that, if the mentioned GoodCloseContacts revision is checked only for equality and not for periodic equivalence of relevant atoms, unrealistic valences of oxygen and silicon atoms are generated.

There can be several configurations which originate these problems, and example of which is illustrated in Figure 4. In the left part of Figure 4 it can be seen that, when more reactive centers are in close proximity, we can see some "collision" possibility if more reactive centers is in proximity. For example, suppose that the $\mathrm{O}-\mathrm{H}$ contact (i.e., black $\mathrm{O}$ and blue $\mathrm{H}$ ) is shorter than the other $\mathrm{O}-\mathrm{H}$ contact (i.e, green $\mathrm{O}$ and blue $\mathrm{H}$ ). Under this condition, the bond between the blue $\mathrm{O}$ and black $\mathrm{Si}$ is created (red line). If no check of the remaining GoodCloseContacts elements is performed, then also the $\mathrm{Si}-\mathrm{O}$ bond between the green $\mathrm{Si}$ and blue $\mathrm{O}$ is created. If only equality check after creation of the blue $\mathrm{O}$ and black Si bond is performed, no problem arises if the configuration corresponds to the portrayed on the left side of Figure 4. However, if the position of the green molecule is shifted to the periodic image position, then both $\mathrm{O}-\mathrm{H}$ atoms (the green $\mathrm{O}$ and the small blue $\mathrm{H}$ atom) are not equal to any other atom (the black and large blue $\mathrm{H}$ atom, and the black and the blue $\mathrm{O}$ atom), but the small blue $\mathrm{H}$ atom is periodically equivalent to that large one, which is a sufficient condition for creating the $\mathrm{Si}-\mathrm{O}$ bond (blue $\mathrm{O}$ and green $\mathrm{Si}$ atoms) which is interrupted by the periodic cell boundary but can be clearly seen using the InCell viewtype. This situation is nicely illustrated in Figure 5, taken from the real system of Figure 3.

Then, the bonding procedure with respect of the next $\mathrm{O}-\mathrm{H}$ close contact from array GoodCloseContacts which has status equal to 1(or, more precisely, the GoodCloseContacts[\$i][0] element) must be repeated.

After a desired number of bonds (\$MaxNumBond) is reached, or at the end of the GoodCloseContacts array is reached, all sets created and populated in the previous steps must be deleted to release computer memory. Also, renaming of the bridging oxygens (\$01) must be performed, since these atoms must not enter the next \$I loop for creating new $\mathrm{O}-\mathrm{H}$ close contacts.

To give a final overview of the entire procedure, the most critical steps are summarized below using a pseusdocode:

***START OF THE SCRIPT***

\#Loading of the structure (*.xsd)

my \$lterMax=50;

\#number of the main loops 
my \$MaxNumBond=3; $\quad$ \#maximum number of created bonds in one I loop

\#Main loop

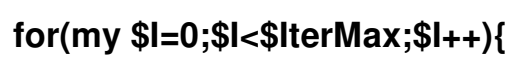

Loading of the setup file (*.xms)

Geometry Optimisation

Annealing

Molecular Dynamics

Creation of the reactive atom arrays and sets

Close contacts calculation

Creation of GoodCloseContacts array and close contacts filtering

Ordering of the GoodCloseContacts elements by increasing close contact length

for (my $\$ \mathrm{i}=0 ;((\$ N u m O f C r e a t e d B o n d s<\$ M a x N u m B o n d) \& \&(\$ i<=\$ \# G o o d C l o s e C o n t a c t s)) ; \$++)\{$

Identification of all the atoms \$O, \$H, \$H1, \$O1, \$Si and \$Si1

Creation of $\$$ Si-\$01 bond

Addition of atoms $\$ \mathrm{O}, \mathbf{\mathrm { H }}, \mathbf{\mathrm { H } 1}$ to array AtomsToDelete

GoodCloseContacts array revision

\} \# end of the \$I loop (Step 4)

\#Deletion of reacted atom sets

\$reactive_O_set->Delete;

\$reactive_H_set->Delete;

\#Deletion of all calculated close contacts

\$CloseContacts->Delete;

\#Creation of the set \$AtomsToDelete_Set from the array AtomsToDelete and appropriate deletion of all the atoms marked in previous steps for deletion

\#the if statement excludes attempt to create set from the empty array

if(\$\#AtomsToDelete $+1>=1)\{$

my \$AtomsToDelete_Set=\$doc->CreateSet("AtomsToDelete",|@AtomsToDelete);

\$AtomsToDelete_Set->Items->Delete;

\}

\#Renaming of oxygen atoms involved in the formation of Si-O-Si bonds (i.e., accessed using the variable \$01)

from "O" to "RO"

foreach my \$atom (@\$atoms \})

\{

if(\$atom->Name eq "O")\{

my $\$$ NumOfSi=0;

foreach my \$atom1 (@\{\$atom->AttachedAtoms\})

\{

if(\$atom1->Name eq "Si")\{\$NumOfSi++;\}

\}\#end \$atom1

if(\$NumOfSi==2)\{\$atom->Name="RO";

\} 
\#end of if(\$atom->Name eq "O"

\}

\#end of loop \$atom

\}

\#end of $\mathbf{I}$ loop (main loop, which should be repeated \$lterMax times)

***END OF THE SCRIPT***

At the very end it may be worth noticing that, despite we release all computer memory properly in each $\mathbf{\$ I}$ loop, during each script run the memory used by the computer is slowly and progressively increasing, so that, after a number of \$I loops, the limit of accessible memory is reached and calculations have to be manually restarted. The number of successfully accomplished \$I loops clearly depends upon the available RAM of a given computer, but also on the amount of close contacts which are created during each \$I loop. That is the reason why we increase MaxAbsoluteDistance from 3 to $6 \AA$.

\subsection{D network relaxation for thermophysical properties determination}

After the GPTMS 3D network structures were built starting from the two partial charge scheme and applying the script detailed above (i.e., two structures I and two structures II), each system was subjected to further annealing cycles up to $600 \mathrm{~K}$. The minimum energy structure for each system was selected for further NVT and NPT molecular dynamics simulation at $300 \mathrm{~K}$ for data collection. The velocity Verlet algorithm was used for integration in all MD simulations. A time step of $0.2 \mathrm{fs}$, and the Nosè/Berendsen thermostat were employed for NPT and NVT MD simulations, respectively. The cut-off for non-bond interactions was set at $9.50 \AA$. The final density of the MD equilibrated systems corresponding to the two different atomic partial charge schemes, AM1-ESP and COMPASS, respectively, is reported in Table 1. As can be seen from Table 1, the equilibrated network densities obtained from initial structure modeled with both partial charge schemes are utterly similar; further, starting from two different annealed initial GTPMS 3D boxes(i.e., structure I and structure II), leads to the same network final density values, thus validating the initial structure selection protocol.

\subsection{Mechanical properties (elastic constants) determination}

The mechanical behavior of a given molecular system can be described by using continuum mechanics. Since, however, any molecular system has a discrete structure, the model to be employed for the estimation of the elastic constants is an equivalent-continuum model (27), in which the overall mechanical response of representative volume elements to an applied set of boundary conditions is equivalent to the response of the molecular representative volume system subjected to the same set of boundary conditions. The equivalentcontinuum is assumed to have a linear-elastic constitutive behavior. The generalized constitutive equation of the equivalent continuum hence is given by:

$\sigma_{i j}=C_{i j k l} \varepsilon_{k l}$

where $\sigma_{i j}$ are the components of the stress tensor $(i, j=1,2,3), C_{i j k l}$ are the components of the linear-elastic stiffness tensor, and $\varepsilon_{k l}$ are the components of the strain tensor. It is further assumed that the system has isotropic material symmetry.

In atomistic calculation, the internal stress tensor in a system can be obtained using the virial expression: 
$\sigma=-\frac{1}{V_{0}}\left[\left(\sum_{i=1}^{N} m_{i}\left(\mathbf{v}_{i} \mathbf{v}_{i}^{T}\right)\right)+\left(\sum_{i<j} r_{i j} \mathbf{f}_{i j}^{T}\right)\right]$

where index $i$ runs over all particles through $N, m_{i}$ and $\mathbf{v}_{i}$ are the mass and the velocity of the particle, respectively, $\mathbf{f}_{i}$ is the force acting on the particle, and $V_{0}$ is the undeformed system volume.

Basically, two classes of methods for calculating material elastic constants using molecular simulations are available in literature at the present. Consistently, the static method (i.e., based on molecular mechanics) was found to be more practical and reliable than the one based on molecular dynamics (28). Thus, a constant strain minimization method, belonging to the class of static methods, was applied to the equilibrated 3D O/I network system. Accordingly, after an initial system energy minimization, three tensile and three pure shear small deformations (to remain within elastic limits) are applied. The system is then again energy minimized following each deformation. The stiffness matrix is calculated from the second derivative of the potential energy $U$ with respect to strain $\varepsilon$ as follows:

$C_{i j}=\frac{1}{V} \frac{\partial^{2} U}{\partial \varepsilon_{i} \partial \varepsilon_{j}}=\frac{\partial \sigma_{i}}{\partial \varepsilon_{j}}=\frac{\sigma_{i+}-\sigma_{i-}}{2 \varepsilon_{j}}$

where $\sigma_{i}$ is the $i$ th component of the internal stress tensor, and $\sigma_{i+}$ and $\sigma_{i-}$ are the components associated with the stress tensor under tension and compression, respectively (further details on the static method are available in (29)). The Lamé constants for the structure $-\lambda$ and $\mu$ - can in turn be calculated from the related stiffness matrix:

$\lambda=\frac{1}{3}\left(C_{11}+C_{22}+C_{33}\right)-\frac{2}{3}\left(C_{44}+C_{55}+C_{66}\right)$

$\mu=\frac{1}{3}\left(C_{44}+C_{55}+C_{66}\right)$

For isotropic materials, the stress-strain behavior can be finally be described in terms of the Lamé constants according to the equations:

$$
\begin{aligned}
& E=\mu \frac{3 \lambda+2 \mu}{\lambda+\mu} \\
& G=\mu \\
& B=\lambda+\frac{2}{3} \mu \\
& v=\frac{\lambda}{2(\lambda+\mu)}
\end{aligned}
$$

where $E, G, B$, and $v$ represent Young's modulus, shear modulus, bulk modulus and Poisson's ratio, respectively.

\subsection{Constant pressure and constant volume heat capacities determination}

Statistical fluctuations about the mean values of quantities measured during the course of an MD simulation can be directly related to thermodynamic properties. Beside common average quantities like density, pressure, 
or energy, the analysis of fluctuations allows to determine properties like heat capacities, compressibility, thermal expansion coefficient, or the Joule-Thomson coefficient. Specific heat capacity at constant pressure $C_{P}$, for instance, is obtained from the fluctuations of energy in the isothermal-isobaric ensemble (NPT) according to:

$C_{P}=\frac{1}{k T^{2}}\left\langle\partial(K+U+p V)^{2}\right\rangle$

where $k$ is the Boltzmann constant, $K$ and $U$ denote the instantaneous values of the kinetic and potential energy, respectively, and $T, P$, and $V$ are the familiar thermodynamic state variables. In addition, the notation $\delta X$ stands for $X-<X>$, where $<X>$ denotes the ensemble average value of a given quantity $X$.

\section{Results and discussion}

\subsection{Structural analysis}

Figure 6 shows the geometry optimized molecular model of the GPTMS molecule, along with the atom labeling used in this work. For this monomer, the relevant structural parameters are listed in Table 2. These include the $\mathrm{Si}-\mathrm{O}$ and $\mathrm{Si}-\mathrm{C} 1$ bond lengths, and the $\mathrm{O}-\mathrm{Si}-\mathrm{O}$ and $\mathrm{O}-\mathrm{Si}-\mathrm{C}$ bond angles, which were estimated to be $1.670 \AA, 1.909 \AA, 108.4^{\circ}$, and $109.5^{\circ}$, respectively. Unfortunately, experimental X-ray diffraction or other spectroscopy data for GPTMS are not available to date. Therefore, to further validate the geometrical features of our model we applied the same model building/optimization procedure to structurally related molecules, for which such information could be retrieved from the literature. The relevant names, structural formulas and geometrical parameters are listed in Table 3.

By comparing the numbers in Tables 2 and 3, given the differences in molecular species and the fact that, whilst ff calculations are performed on an isolated molecule in vacuum the corresponding experimental quantities are obtained either from single-crystal or spectroscopic studies, we can conclude that all our results are in good agreement with the literature data.

In order to check the influence of the atomic partial charges on the final properties of the system, the GPTMS optimized structure was also assigned a partial charge distribution as derived from AM1-ESP calculations (see Table 4). Generally, speaking, a common procedure to estimate atomic partial charges is via Mulliken analysis (33). However, since the results may be strongly dependent on the basis set employed, and there is no unambiguous method to assign charge to two atoms within a given bond (34), we tried to bypass the problem by fitting the point charges at preselected positions to the electrostatic potential surface (ESP) $(35,36)$. On the other hand, the partial charge scheme thus obtained may be, in turn, dependent on the specific molecular conformation considered (37). To verify whether this was our case, we tested the obtained charge distribution by calculating molecular dipole moments and standard enthalpies of formation for all molecules listed in Table 3, and compared them with the corresponding experimental values, where available (see Table 5). As results from this Table, once again the agreement between calculate and experimental quantities is good, thus confirming the validity of the adopted approach.

According to the procedure outline above, an hybrid O/I 3D system based on GPTMS was successfully generated with high conversion. It is well known that a $100 \%$ conversion is rarely achieved experimentally because of gel transition at later stage. Although other systems with a different (lower) conversion degree $\alpha$ could be generated by changing, for instance, the distance between close contacts up to a reasonable value of $10 \AA$, the amount of unreacted group in the actual simulated molecular systems is, on average, equal to $10 \%$, yielding $\alpha=0.9$. 
The central unit cells of the initial GPTMS monomers, and the final network systems obtained from both structures I and II are shown in Figures 4, 5 and 6, respectively. As can be seen from Figures 5 and 6, the 3D network structures is characterized by the presence of both chemical and physical crosslinks. Some bonds clearly connect to image cells across the boundary, and thus extend throughout the periodic system. Due to the scarcity of experimental characterization, which by the way accounts for the small number of simulation studies on crosslinked polymeric systems (39-41), only a few comparisons between experimental and simulation can be attempted based on the main structural features of the systems.

As mentioned in the introduction, PSSQOs are the generic products obtained by the hydrolytic condensation of monomers such as GPTMS. Strictly speaking, the term refers to fully condensed structures of formula $\left(\mathrm{RSiO}_{3 / 2}\right)_{\mathrm{n}}(\mathrm{n}=$ even number$)$, also denoted $T_{\mathrm{n}}$. But the term is frequently extended to denote partially condensed structures, $\left[\mathrm{RSiO}_{3 / 2-\mathrm{x}}(\mathrm{OH})_{2 \mathrm{x}}\right]_{\mathrm{m}}$ or $T_{\mathrm{n}}(\mathrm{OH})_{\mathrm{m}}$, where $\mathrm{m}=0,2,4 \ldots(2+\mathrm{n})$ for $\mathrm{n}=$ even number and $\mathrm{m}=$ $1,3,5 \ldots(2+n)$ for $n=$ odd number. Keeping this definition, PSSQO structures may vary from perfect polyhedra, incompletely condensed polyhedral, ladder polymers, open structures, linear polymers, and all possible combinations thereof. Generally speaking, then, according to the polymerization conditions employed different structures for the resulting networks are proposed, such as randomly connected threedimensional networks of trifunctional monomers, "ladder" structures, and a combination of linear, "ladder" and cage-like fragments (42).

As the presence and relative amount of the structures described above significantly affect the network final structure, homogeneity and, ultimately, the mechanical properties of the resulting hybrids, the different reaction steps can be optimized to obtain final materials with targeted properties. Experimentally, it has been found that, under acid conditions, only a very small amount of cage-like structures are formed in the GPTMS polymerization, the intermolecular condensation being the preferred mechanism of network growth (6). Accordingly, a high-molecular weight branched PSSQO grows, until a gel system is formed at a conversion degree approximately equal to 0.7 . The corresponding composition of the gel state exhibits a considerable amount of silicon atoms involved in triple intermolecularly branched units representing the cross-links in the network. Further SAXS experiments revealed also that, under acid catalysis, no microphase separation resulting in self-assembly of regularly arranged domains is present (6). Contrarily, a broad distribution of high-molecular weight PSSQOs with dangling organic substituents is formed, the structure not promoting any ordering. The inspection of the 3D structures obtained from our simulation procedure compare well with the network picture described above. Indeed, the overall network structure is linear and extends in all three directions, the presence of unstrained rings with a number of Si atoms greater than 4 are present. To further confirm the quality of the network structures, Table 6 lists the mean values of some geometrical parameters, as obtained from initial structure I with the COMPASS atomic partial charges scheme by averaging over 10 MD frames. By comparing these data with the corresponding geometrical features of the GPTMS monomer model reported in Table 2, we can see that the differences are rather small, indeed confirming that crosslinking does not result in considerable geometrical modifications, and no substantial strain is induced in the final network.

\subsection{Thermophysical properties of the hybrid O/I network}

\subsubsection{Mechanical properties (elastic constants)}

The mechanical properties calculated using the 3D network structures obtained from the MD simulations are reported in Table 7 . The second column refers to the properties of the network structures simulated with the partial charge scheme obtained using the AM1-ESP approach, whilst the third column lists the same quantities resulting from the 3D structures bearing the partial charges as assigned by the COMPASS ff (see Table 4). In 
the last column of this Table, the range of experimental values of the corresponding mechanical properties $(43,44)$ is also reported for comparison.

The data in Table 7 indicate that the charging method has a certain impact on the mechanical properties of the final material. Indeed, in the case of the AM1-ESP partial charging scheme the values obtained from the simulation are located close to the lower limit of the expected range, if not below. With the COMPASS force field charge scheme, the mechanical characteristic of the GPTMS network lay in the expected range. Although no experimental measures are available for our systems, as a conclusion we could say that the predictions obtained by using a standard force field satisfactorily reproduce the available mechanical experimental data.

\subsubsection{Heat capacities}

The specific heat capacities at constant volume and constant pressure, $c_{v}$ and $c_{P}$, calculated for the GPTMSbased $\mathrm{O} / \mathrm{I}$ network are reported in Table 8. Again, these properties were obtained for structure bearing the two different partial charge schemes (see second and third column of Table 8 for AM1-ESP and COMPASS charge scheme, respectively). In the last column, the experimental data range available in literature for similar systems is also shown for comparison $(43,44)$.

As seen for the mechanical properties, all data calculated starting from structures bearing atomic partial charges derived from the AM1-ESP approach lay in the lower limit of the experimental data range, whilst those obtained from the COMPASS partial charge set fall better within the interval of observed values.

\section{Conclusions}

In this work a computational procedure, based on a Perl script developed in Materials Studio v. 4.1, is presented, which allowed to obtain atomistic models of hybrid organic/inorganic networks based on GPTMS. The actual procedure, however, can be applied with minor modifications to any similar system. Furthermore, the translation of the script in other programming languages (e.g., $\mathrm{C}^{++}$Fortran, etc.) makes this script platform independent and, coupled to an MD routine, allows the same scientific results to be achieved with other simulation codes. The simulations allowed also to estimate several thermophysical properties of the 3D network, such as elastic constants and specific heats, whose values were found to be in the range of the corresponding experimental evidence for similar systems. These results confirm both the accuracy of the 3D model structure generated by the script procedure, and the quality of the force field used. Therefore, the proposed computational recipe can constitute a useful tool for the design and development of new hybrid O/I systems with specific structural/chemico-physical properties.

\section{Acknowledgements}

This work was carried out in the frame of the European Union Research Framework Programme FP6 "MultiPro". All authors gratefully acknowledge the EU for the generous financial support. M.M. received further support from the Ministry of Education of Czech Republic (Project LC 06041) and by AS CR (project KAN 1011 20701). All authors wish to thank Dr. Martin Lísal for insightful comments. Special thanks are also due to Carsten Menke, James Wescott and Stephen Todd from Accelrys, for their valuable help in solving several technical issues, and for useful discussion. 


\section{References}

1. Sanchez, C.; Soler-Illia, G.J.d.A.A.; Ribot, F.; Lalot, T.; Mayer, C.R.; Cabuil, V. Chem. Mater. 2001, 13, 3061.

2. Livage, J.; Sanchez, C. J. Non-Cryst. Solids 1992, 145, 11.

3. Kelts, L.W.; Armstrong, N.J. J. Mater. Res. 1989, 4, 423.

4. Mateika, L.; Dukh, O.; Brus, J.; Simonsick, W.J.Jr.; Meissner, B. J. Non-Cryst. Solids 2000, 270, 34.

5. Eisenber, P.; Erra-Balsells, R.; Ishikawa, Y.; Lucas, J.C.; Mauri, A.N.; Nonami, H.; Riccardi, C.C.; Williams, R.J.J. Macromolecules 2000, 33, 1940.

6. Matejka, L.; Dukh, O.; Hlavata, D.; Meissner, B.; Brus, J. Macromolecules 2001, 34, 6904.

7. Brus, J.; Spirkova, M.; Hlavata, D.; Strachota, A. Macromolecules 2004, 37, 1346.

8. Nass, R.; Arpac, E.; Glaubitt, W.; Schmidt, H. J. Non-Cryst. Solids 1990, 121, 370.

9. Philipp, G.; Schmidt, H. J. Non-Cryst. Solids 1984, 63, 283.

10. Popall, M.; Kappel, J.; Pilz, M.; Schulz, J.; Feyder, G. J. Sol-Gel Sci. Technol. 1994, 2, 157.

11. Schmidt, H. J. Non-Cryst. Solids 1994, 178, 302.

12. Sorek, Y.; Zevin, M.; Reisfeld, R.; Hurvits, T.; Rushin, S. Chem. Mater. 1997, 9, 670.

13. Knobbe, E.T.; Dunn, B.; Fuqua, P.D.; Nishida, F. Appl. Opt. 1990, 29, 2729.

14. Sorek, Y.; Reisfeld, R.; Tenne, R. Chem. Phys. Lett. 1994, 227, 235.

15. (a) Guglielmi, M.; Brusatin, G.; Della Giustina G. J, Non-Cryst. Solids 2007, 353, 1681. (b) Della Giustina, G.; Brusatin, G.; Guglielmi M.; Romanato F. Mat. Sci. Eng. C 2007, 27, 1382. (c) Brusatin, G.; Della Giustina, G.; Guglielmi, M.; Casalboni, M.; Prosposito, P.; Schutzmann S.; Roma G. Mat. Sci. Eng. C 2007, 27, 1022.

16. Zevin, M.; Reisfeld, R. Opt. Mater. 1997, 8, 37.

17. Sun, H.; Rigby, D. Spectrochim. Acta Part A 1997, 53, 1301.

18. Sun, H. Macromolecules 1995, 28, 701.

19. Brusatin, G., personal communication.

20. Scocchi, G.; Posocco, P.; Fermeglia, M.; Pricl, S. J. Phys. Chem. B. 2007, 111, 2143.

21. Fermeglia, M.; Cosoli, M.; Ferrone, M.; Piccarolo, S.; Mensitieri, G.; Pricl, S. Polymer 2006, 47, 5979.

22. Fermeglia, M.; Ferrone, M.; Pricl, S. Mol. Simulation 2004, 30, 289.

23. Toth, R.; Coslanich, A.; Ferrone, M.; Fermeglia, M.; Pricl, S.; Miertus, S.; Chiellini, E. Polymer 2004, 45, 8075 .

24. Fermeglia, M.; Ferrone, M.; Pricl, S. Fluid Phase Equilib. 2003, 212, 315.

25. Fermeglia, M.; Pricl, S. AIChE J. 1999, 45, 2619.

26. Fidalgo, A.; Ilharco, L.M. J. Non-Cryst. Solids 2001, 283, 144.

27. Odegard, G.M.; Gates, T.S.; Nicholson, L.M.; Wise, K.E. Compos. Sci. Technol. 2002, 62, 1869.

28. Raaska,T.; Niemela, J.S.; Sundholm, F. Macromolecules 1994, 27, 5751.

29. Theodorou, D.N.; Suter, U.W. Macromolecules 1986, 191, 139.

30. Handbook of Chemistry and Physics, Lide D.R. Ed., $79^{\text {th }}$ ed., CRC Press, Boca Raton, FL, USA, 1999

31. Barrow, M.J., Ebsworth, E.A., Harding, M.M. Acta Crystal. 1979, B35, 2091.

32. Koput, J., Wierzbicki, A. J. Mol. Spectrosc. 1983, 99, 116.

33. Mulliken, R.S. J. Chem. Phys. 1955, 23, 1833.

34. Singh, U.C.; Kollman, P.A. J. Comput. Chem. 1984, 5, 129.

35. Weiner, S.J.; Kolmann, P.A.; Nguyen, D.T.; Case, D.A. J. Comput. Chem. 1986, 7, 230. 
36. Weiner, S.J.; Kolmann, P.A.; Case, D.A.; Singh, U.C.; Ghio, C.; Alagona, G., Profeta, S.; Weiner, P.J. J. Am. Chem. Soc. 1984, 106, 765.

37. Reynolds, C.A.; Essex, J.W.; Richards, W.J. J. Am. Chem. Soc. 1992, 114, 9075.

38. Girard-Reydet, E.; Riccardi, C.C.; Sautereau, H.; Pascault, J.P. Macromolecules 1995, $28,7599$.

39. Fan, H.B.; Yuen, M.M.F. Polymer 2007, 48, 2174.

40. Wu, C.; Xu, W. Polymer 2006, 47, 6004.

41. Yarowsky, I.; Evans, E. Polymer 2002, 43, 963.

42. Boury, B.; Corriu, R. Chem. Rec. 2003, 3, 120.

43. Van Krevelen, D.W. Properties of polymers, Elsevier, Amsterdam, The Netherlands (1990).

44. Bicerano, J. Prediction of polymers properties, M. Dekker, New York, USA (1996). 


\section{Page 19 of 39}

1

2

3

4

5

6

7

8

10

11

12

13

14

15

16

17

18

19

20

2

22

23

24

25

26

27

28

29

30

34

35

36

37

38

39

40

41

42

43

44

45

46

47

48

49

50

51

52

53

54

55

56

57

58

59

60

http://mc.manuscriptcentral.com/tandf/jenmol 


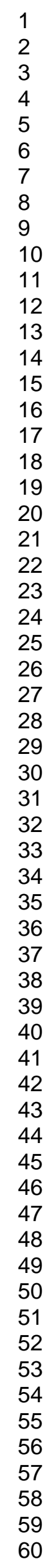




\section{Page 21 of 39}

1

2

3

4

5

6

7

8

10

11

13

14

15

16

17

18

19

20

21

22

23

24

25

26

27

28

29

30

31

32

33

34

35

36

37

38

39

40

41

42

43

44

45

46

47

48

49

50

51

52

53

54

55

56

57

58

59

60
Table 1. MD equilibrated density values $\rho$ for the 3D GPTMS-based O/I network structures obtained from the simulations.

\begin{tabular}{lll}
\hline & Initial structure I & Initial structure II \\
Atomic partial charge scheme & $\rho\left(\mathrm{g} / \mathrm{cm}^{3}\right)$ & $\rho\left(\mathrm{g} / \mathrm{cm}^{3}\right)$ \\
\hline AM1-ESP & 1.368 & 1.369 \\
COMPASS & 1.371 & 1.368 \\
\hline
\end{tabular}


Table 2. Geometrical parameters of the GPTMS molecular model after geometry optimization with COMPASS ff. Atom numbering as in Figure 6.

\begin{tabular}{|c|c|c|c|c|c|c|c|c|c|c|c|}
\hline $\begin{array}{l}\text { Si- } \\
\mathrm{O} 1(\mathrm{O} 2, \mathrm{O} 3) / \AA\end{array}$ & $\mathrm{Si}-\mathrm{C} 1 / \AA$ & $\begin{array}{l}\mathrm{H} 1- \\
\mathrm{O} 1 / \AA\end{array}$ & C1-C2/Å & $\mathrm{C} 2-\mathrm{C} 3 / \AA$ & C3-O4/A & $\mathrm{O} 4-\mathrm{C} 4 / \AA$ & C4-C5/Å & $\begin{array}{l}\text { C5(C6)- } \\
\text { O5/A }\end{array}$ & C5-C6/Å & H4-C1/Å & $\begin{array}{l}\mathrm{H} 12- \\
\mathrm{C} 5 / \AA\end{array}$ \\
\hline 1.670 & 1.909 & 1.110 & 1.531 & 1.527 & 1.419 & 1.419 & 1.495 & 1.428 & 1.439 & 1.104 & 1.098 \\
\hline O1-Si-O2 & $\begin{array}{l}\mathrm{H} 1-\mathrm{O} 1- \\
\mathrm{Si}\end{array}$ & $\begin{array}{l}\text { Si-C1- } \\
\mathrm{C} 2\end{array}$ & $\begin{array}{l}\text { C1-C2- } \\
\text { C3 }\end{array}$ & $\begin{array}{l}\mathrm{C} 2-\mathrm{C} 3- \\
\mathrm{O} 4\end{array}$ & $\begin{array}{l}\mathrm{C} 3-\mathrm{O} 4- \\
\mathrm{C} 4\end{array}$ & $\begin{array}{l}\text { O4-C4- } \\
\text { C5 }\end{array}$ & $\begin{array}{l}\text { C4-C5- } \\
\text { C6 }\end{array}$ & C5-O5-C6 & $\mathrm{H} 6-\mathrm{C} 2-\mathrm{H} 7$ & $\begin{array}{l}\text { H13-C6- } \\
\text { H14 }\end{array}$ & \\
\hline $108.4^{\circ}$ & $109.5^{\circ}$ & $114.7^{\circ}$ & $115.1^{\circ}$ & $106.6^{\circ}$ & $114.2^{\circ}$ & $105.9^{\circ}$ & $125.4^{\circ}$ & $60.2^{\circ}$ & $107.2^{\circ}$ & $112.2^{\circ}$ & \\
\hline
\end{tabular}




\section{Page 23 of 39}

Table 3. Computed geometrical data for molecules structurally related to GPTMS. Experimental available data (30-32) are reported in parenthesis for comparison.

\begin{tabular}{|c|c|c|c|c|c|c|c|c|}
\hline Name & Chemical & Chemical & & & etrical par & meters & & \\
\hline Dimethyl ether & $\mathrm{C}_{2} \mathrm{H}_{6} \mathrm{O}$ & & $\begin{array}{l}\mathrm{C}-\mathrm{O} / \AA \\
1.412 \\
(1.416)\end{array}$ & $\begin{array}{l}\mathrm{C}-\mathrm{H} / \AA \\
1.124^{\circ} \\
\left(1.121^{\circ}\right)\end{array}$ & $\begin{array}{l}\mathrm{C}-\mathrm{O}-\mathrm{C} \\
111.8^{\circ} \\
\left(112^{\circ}\right)\end{array}$ & $\begin{array}{l}\mathrm{H}-\mathrm{C}-\mathrm{C} \\
108.3^{\circ} \\
\left(108^{\circ}\right)\end{array}$ & & \\
\hline Ethylene oxide & $\mathrm{C}_{2} \mathrm{H}_{4} \mathrm{O}$ & & $\begin{array}{l}\text { C-C/A } \\
1.459 \\
(1.466)\end{array}$ & $\begin{array}{l}\text { C-H/A } \\
1.096 \\
(1.085)\end{array}$ & $\begin{array}{l}\mathrm{C}-\mathrm{O} / \AA \\
1.428 \\
(1.431)\end{array}$ & $\begin{array}{l}\mathrm{C}-\mathrm{O}-\mathrm{C} \\
60.4^{\circ}\end{array}$ & $\begin{array}{l}\text { C-C-H } \\
116.5^{\circ} \\
\left(116.6^{\circ}\right)\end{array}$ & \\
\hline Propylene oxide & $\mathrm{C}_{3} \mathrm{H}_{6} \mathrm{O}$ & & $\begin{array}{l}\mathrm{C}\left(\mathrm{H}_{3}\right)- \\
\mathrm{C}(\mathrm{H}) / \AA \\
1.527 \\
(1.510)\end{array}$ & $\begin{array}{l}\mathrm{C}(\mathrm{H})- \\
\mathrm{C}\left(\mathrm{H}_{2}\right) / \AA \\
1.452\end{array}$ & $\begin{array}{l}\mathrm{C}-\mathrm{O} / \AA \\
1.426\end{array}$ & $\begin{array}{l}\mathrm{C}-\mathrm{O}-\mathrm{C} \\
60.4^{\circ}\end{array}$ & $\begin{array}{l}\text { C-C-C } \\
122^{\circ} \\
\left(121^{\circ}\right)\end{array}$ & \\
\hline $\begin{array}{l}\text { Propyl Methyl } \\
\text { Ether }\end{array}$ & $\mathrm{C}_{4} \mathrm{H}_{10} \mathrm{O}$ & & $\begin{array}{l}\mathrm{C}-\mathrm{O} / \AA \\
1.415 \\
(1.418)\end{array}$ & $\begin{array}{l}\mathrm{C}-\mathrm{C} / \AA \\
1.527 \\
(1.520)\end{array}$ & $\begin{array}{l}\text { C-H/A } \\
1.115 \\
(1.118)\end{array}$ & $\begin{array}{l}\text { C-O-C } \\
112.3^{\circ} \\
\left(111.9^{\circ}\right)\end{array}$ & $\begin{array}{l}\text { O-C-C } \\
109.7^{\circ} \\
\left(109.4^{\circ}\right)\end{array}$ & $\begin{array}{l}\text { H-C-H } \\
109.2^{\circ} \\
\left(109^{\circ}\right)\end{array}$ \\
\hline Disiloxane & $\mathrm{H}_{6} \mathrm{OSi}_{2}$ & & $\begin{array}{l}\mathrm{Si}-\mathrm{O} / \AA \\
1.635 \\
(1.63)^{\mathrm{a}}\end{array}$ & $\begin{array}{l}\mathrm{Si}-\mathrm{H} / \AA ̊ \\
1.485 \\
(1.490)\end{array}$ & $\begin{array}{l}\text { Si-O-Si } \\
151.3^{\circ} \\
\left(151.2^{\circ}\right)^{b}\end{array}$ & $\begin{array}{l}\mathrm{H}-\mathrm{Si}-\mathrm{H} \\
109.8^{\circ}\end{array}$ & & \\
\hline $\begin{array}{l}\text { Hexamethyl- } \\
\text { disiloxane }\end{array}$ & $\mathrm{C}_{6} \mathrm{H}_{18} \mathrm{OSi}_{2}$ & & $\begin{array}{l}\mathrm{Si-O} / \AA \\
1.639 \\
(1.638)\end{array}$ & $\begin{array}{l}\mathrm{Si}-\mathrm{C} / \AA \\
1.862 \\
(1.869)\end{array}$ & $\begin{array}{l}\mathrm{H}-\mathrm{C} / \AA \AA \\
1.101 \\
(1.104)\end{array}$ & $\begin{array}{l}\text { C-Si-O } \\
108.3^{\circ} \\
\left(109.0^{\circ}\right)\end{array}$ & $\begin{array}{l}\text { Si-O-Si } \\
149.7^{\circ} \\
\left(151.3^{\circ}\right)\end{array}$ & $\begin{array}{l}\text { C-Si- } \\
\mathrm{C} \\
110.8^{\circ}\end{array}$ \\
\hline Methoxysilane & $\mathrm{CH}_{6} \mathrm{OSi}$ & & $\begin{array}{l}\mathrm{Si}-\mathrm{C} / \AA \\
1.421\end{array}$ & $\begin{array}{l}\mathrm{Si-O} / \AA \\
1.669\end{array}$ & $\begin{array}{l}\mathrm{H}-\mathrm{C} / \AA \\
1.101\end{array}$ & $\begin{array}{l}\mathrm{H}-\mathrm{Si} / \AA ̊ \\
1.476\end{array}$ & $\begin{array}{l}\mathrm{C}-\mathrm{O}-\mathrm{Si} \\
122.6^{\circ}\end{array}$ & $\begin{array}{l}\mathrm{H}-\mathrm{Si}- \\
\mathrm{H} \\
110.8^{\circ}\end{array}$ \\
\hline Tetramethylsilane & $\mathrm{C}_{4} \mathrm{H}_{12} \mathrm{Si}$ & & $\begin{array}{l}\text { Si-C/Å } \\
1.880 \\
(1.875)\end{array}$ & $\begin{array}{l}\text { H-C/A } \\
1.110 \\
(1.115)\end{array}$ & $\begin{array}{l}\text { C-Si-C } \\
109.5^{\circ}\end{array}$ & $\begin{array}{l}\text { H-C-H } \\
109.7^{\circ} \\
\left(109.8^{\circ}\right)\end{array}$ & & \\
\hline
\end{tabular}


Table 4. Partial charges on GPTMS atoms as assigned by COMPASS and obtained from AM1-ESP calculations. Atom numbering as in Figure 6.

\begin{tabular}{|c|c|c|c|c|c|c|c|c|c|c|c|c|c|c|}
\hline Atom name & $\mathrm{Si}$ & $O 1$ & $O 2$ & O3 & O4 & O5 & $C 1$ & $C 2$ & $C 3$ & C4 & $C 5$ & C6 & & \\
\hline COMPASS & 0.80 & -0.47 & -0.47 & -0.47 & -0.32 & -0.32 & -0.24 & -0.11 & 0.054 & 0.054 & 0.11 & 0.054 & & \\
\hline AM1-ESP & 0.72 & -0.27 & -0.32 & -0.30 & -0.10 & -0.099 & -0.25 & -0.20 & -0.22 & 0.14 & -0.12 & -0.052 & & \\
\hline Atom name & H1 & $\mathrm{H} 2$ & $\mathrm{H} 3$ & $\mathrm{H} 4$ & H5 & H6 & $\mathrm{H} 7$ & H8 & H9 & H10 & H11 & H12 & H13 & H14 \\
\hline COMPASS & 0.25 & 0.25 & 0.25 & 0.053 & 0.053 & 0.053 & 0.053 & 0.053 & 0.053 & 0.053 & 0.053 & 0.053 & 0.053 & 0.053 \\
\hline AM1-ESP & 0.13 & 0.12 & 0.12 & 0.045 & 0.064 & 0.10 & 0.093 & 0.084 & 0.096 & 0.016 & -0.014 & 0.070 & 0.076 & 0.072 \\
\hline
\end{tabular}


Table 5. Computed dipole moment and standard enthalpy of formation for molecules structurally related to GPTMS. Experimental available data are reported in parenthesis for comparison.

\begin{tabular}{lllll}
\hline Name & $\mu_{\text {calc }}(\mathrm{D})$ & $\mu_{\exp }(\mathrm{D})$ & $\Delta \mathrm{H}_{\text {form,calc }}(\mathrm{kcal} / \mathrm{mol})$ & $\Delta \mathrm{H}_{\text {form,exp }}(\mathrm{kcal} / \mathrm{mol})$ \\
\hline Dimethyl ether & 1.36 & 1.30 & -44.66 & -44.00 \\
Ethylene oxide & 1.95 & 1.89 & -13.61 & -12.57 \\
Propylene oxide & 2.06 & 2.01 & -21.68 & -22.63 \\
Propyl Methyl Ether & 1.13 & 1.11 & -55.08 & -56.91 \\
Disiloxane & 0.34 & 0.24 & -65.22 & - \\
Hexamethyldisiloxane & 0.38 & - & -182.01 & -185.87 \\
Methoxysilane & 1.1 & 1.15 & -53.98 & - \\
Tetramethylsilane & 0.00 & 0.00 & -57.99 & -57.12 \\
\hline
\end{tabular}


Table 6. Mean geometrical parameters of the GPTMS 3D network obtained from structure I with the COMPASS atomic partial charges scheme by averaging over $10 \mathrm{MD}$ frames.

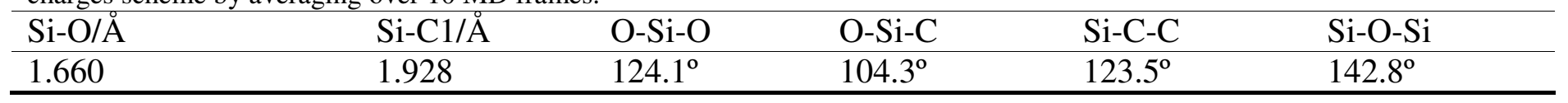




\section{Page 27 of 39}

4

5

6

7

8

9

10

11

12

13

14

15

16

17

18

19

20

21

22

23

24

25

26

27

28

29

30

31

32

33

34

35

36

37

38

39

40

41

42

43

44

45

46

47

48

49

50

51

52

53

54

55

56

57

58

59

60

Table 7. Young modulus $E$, bulk modulus $B$, shear modulus $G$, Poisson ratio $v$, and Lamé constants $\lambda$ and $\mu$ for the 3D GPTMS-based O/I network structures obtained from MD simulations.

\begin{tabular}{llllll}
\hline Mechanical properties & $\begin{array}{l}\text { Structure I } \\
\text { AM1-ESP }\end{array}$ & $\begin{array}{l}\text { Structure II } \\
\text { AM1-ESP }\end{array}$ & $\begin{array}{l}\text { Structure I } \\
\text { COMPASS }\end{array}$ & $\begin{array}{l}\text { Structure II } \\
\text { COMPASS }\end{array}$ & $\begin{array}{l}\text { Experimental } \\
\text { data }^{\mathrm{a}}\end{array}$ \\
\hline$E(\mathrm{GPa})$ & 2.85 & 3.00 & 3.52 & 3.96 & $1.9-4.5$ \\
$B(\mathrm{GPa})$ & 1.89 & 2.00 & 2.58 & 2.66 & $2.5-7$ \\
$G(\mathrm{GPa})$ & 1.14 & 1.20 & 1.38 & 1.58 & $0.9-1.5$ \\
$v$ & 0.25 & 0.27 & 0.27 & 0.25 & $0.3-0.4$ \\
$\lambda(\mathrm{GPa})$ & 1.13 & 1.20 & 1.66 & 1.61 & - \\
$\mu(\mathrm{GPa})$ & 1.14 & 1.20 & 1.38 & 1.58 & - \\
\hline
\end{tabular}

${ }^{\mathrm{a}}$ Average values for crosslinked networks 
1

2

3

4

5

6

7

8

9

10

11

12

13

14

15

16

17

18

19

20

21

22

23

24

25

26

27

28

29

30

31

32

33

34

35

36

37

38

39

40

41

42

43

44

45

46

47

48

49

50

51

52

53

54

55

56

57

58

59

60

Table 8. Specific constant pressure $\left(c_{P}\right)$ heat capacity for the 3D GPTMS-based O/I network structures obtained from the MD simulations.

\begin{tabular}{llllll}
\hline Specific heat capacity & $\begin{array}{l}\text { Structure I } \\
\text { AM1-ESP }\end{array}$ & $\begin{array}{l}\text { Structure II } \\
\text { AM1-ESP }\end{array}$ & $\begin{array}{l}\text { Structure I } \\
\text { COMPASS }\end{array}$ & $\begin{array}{l}\text { Structure II } \\
\text { COMPASS }\end{array}$ & $\begin{array}{l}\text { Experimental } \\
\text { data }^{\mathrm{a}}\end{array}$ \\
\hline$c_{P}(\mathrm{~kJ} / \mathrm{kgK})$ & 1.16 & 1.23 & 1.64 & 1.49 & $1.1-2.2$ \\
\hline
\end{tabular}

${ }^{\mathrm{a}}$ Average values for crosslinked networks 


\section{Figure Captions}

Scheme 1. Reaction leading to the Si-O-Si network formation.

Figure 1. Details of the GPTMS molecular modeling showing, in stick-and-ball representation, the reactive atom pairs (top), and the newly formed Si-O-Si bond and the corresponding deleted water molecule (bottom). All other atoms are in line rendering. Color code: $\mathrm{Si}$, gold; O, red; $\mathrm{C}$, gray, $\mathrm{H}$, white.

Figure 2. Atom/variable association in the bonding scheme considered in this work.

Figure 3. GPTMS crosslinked structure created using only equality (not equivalence) check of the atoms during the GoodCloseContacts revision. The unrealistic oxygen atoms forming three bonds with Si are highlighted in green, whilst the Si atoms with 4 oxygen bonds are highlighted in violet.

Figure 4. Schema of one problematic configuration leading to unrealistic valences of $\mathrm{O}$ and Si atoms (see text for details).

Figure 5. Details of the real system illustrated in Figure 3. Top: InCell lattice display style view; bottom: default lattice display style view.

Figure 6. Geometry optimized molecular model of the GPTMS molecule, along with the atom labeling used in this work. All atoms are in stick-and-ball representation, using the same color code of Figure 1.

Figure 7. Central unit cell of the initial GPTMS monomers. Color code as in Figure 1.

Figure 8. Hybrid O/I 3D network structures obtained starting from GPTMS models bearing atomic partial charges calculated with the AM1-ESP method (structure I, top left, structure II, top right), and assigned by the COMPASS ff (structure I, bottom left, structure II, bottom right). (see text for details). Crosslinked Si and O atoms are highlighted in Stick-and-Ball. Color code as in Figure 1.

Figure 9. Details of the hybrid O/I 3D network structures obtained starting from GPTMS models bearing atomic partial charges calculated with the AM1-ESP method (structure I, top left, structure II, top right), and assigned by the COMPASS ff (structure I, bottom left, structure II, bottom right). (see text for details).

Crosslinked Si and $\mathrm{O}$ atoms are highlighted in Stick-and-Ball. Color code as in Figure 1. 


\section{Page 31 of 39}

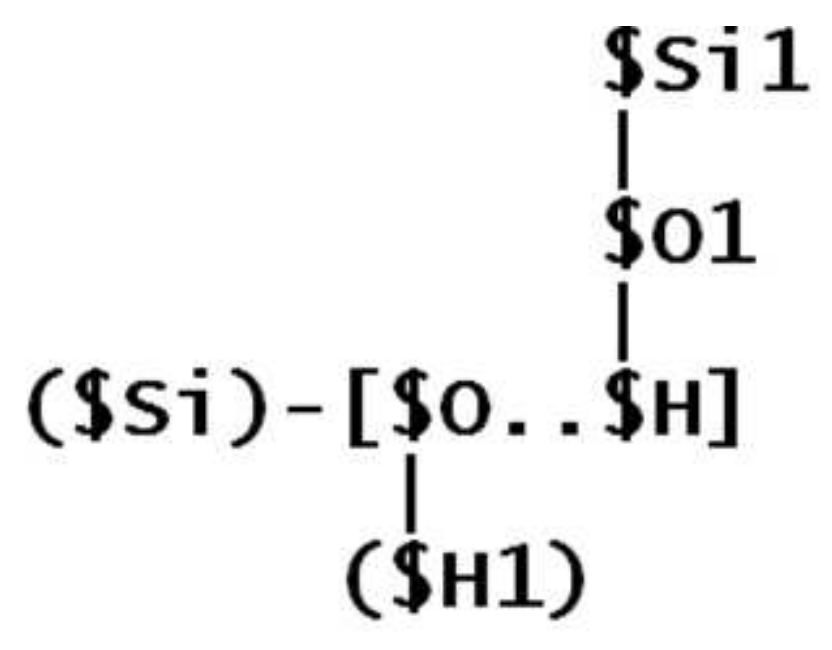

$69 \times 51 \mathrm{~mm}(115 \times 117 \mathrm{DPI})$

http://mc.manuscriptcentral.com/tandf/jenmol 


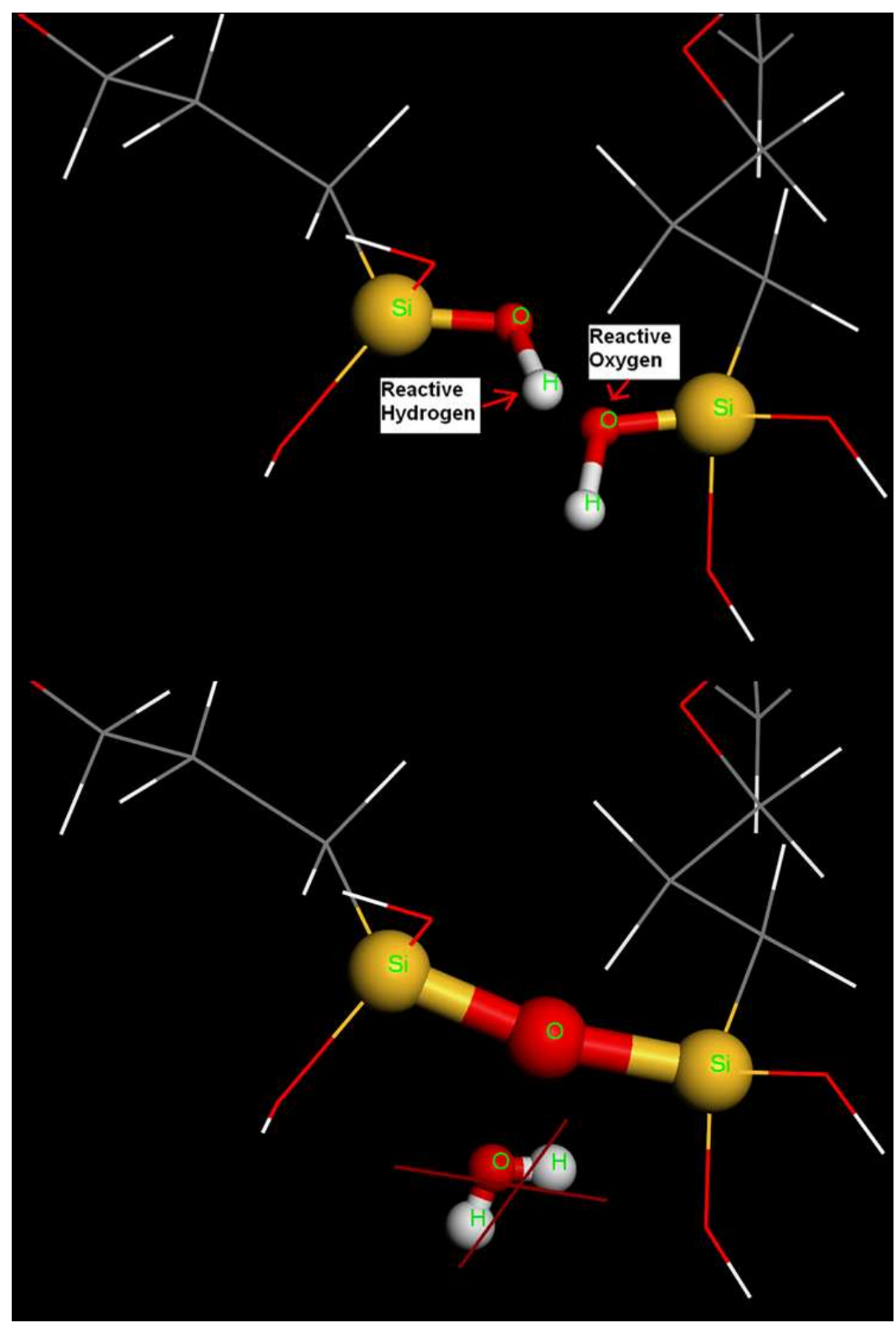

$125 \times 186 \mathrm{~mm}(150 \times 150 \mathrm{DPI})$

http://mc.manuscriptcentral.com/tandf/jenmol 


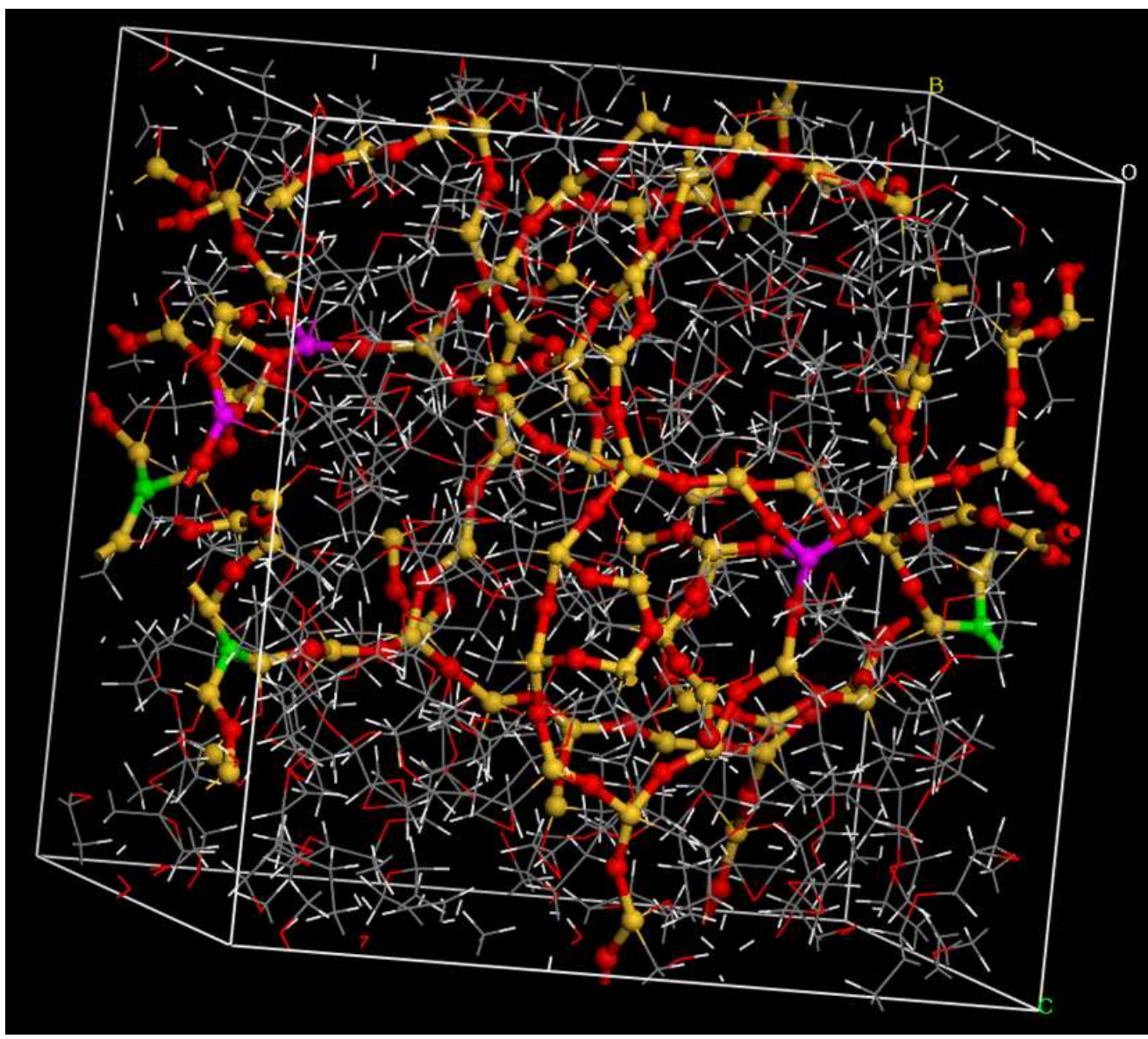

$139 \times 125 \mathrm{~mm}(150 \times 150 \mathrm{DPI})$

http://mc.manuscriptcentral.com/tandf/jenmol 

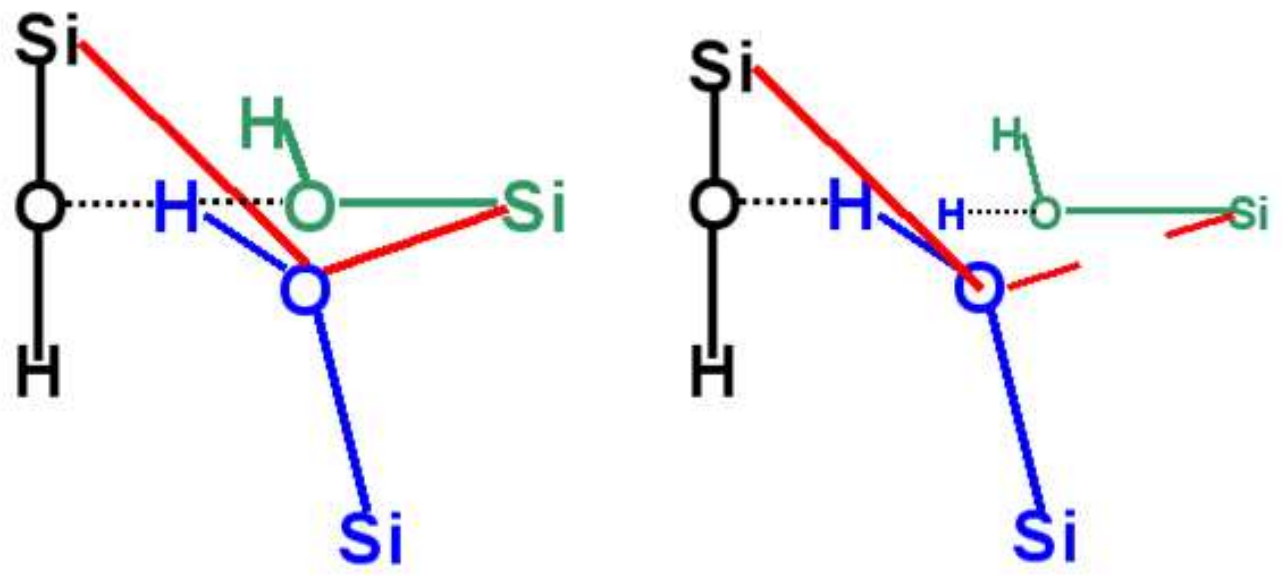

$145 \times 66 \mathrm{~mm}(100 \times 100$ DPI $)$

http://mc.manuscriptcentral.com/tandf/jenmol 


\section{Page 35 of 39}

1
2
3
4
5
6
7
8
9
10
11
12
13
14
15
16
17
18
19
20
21
22
23
24
25
26
27
28
29
30
31
32
33
34
35
36
37
38
39
40
59
50
51
50
43
44
45
47
48
49
50
52

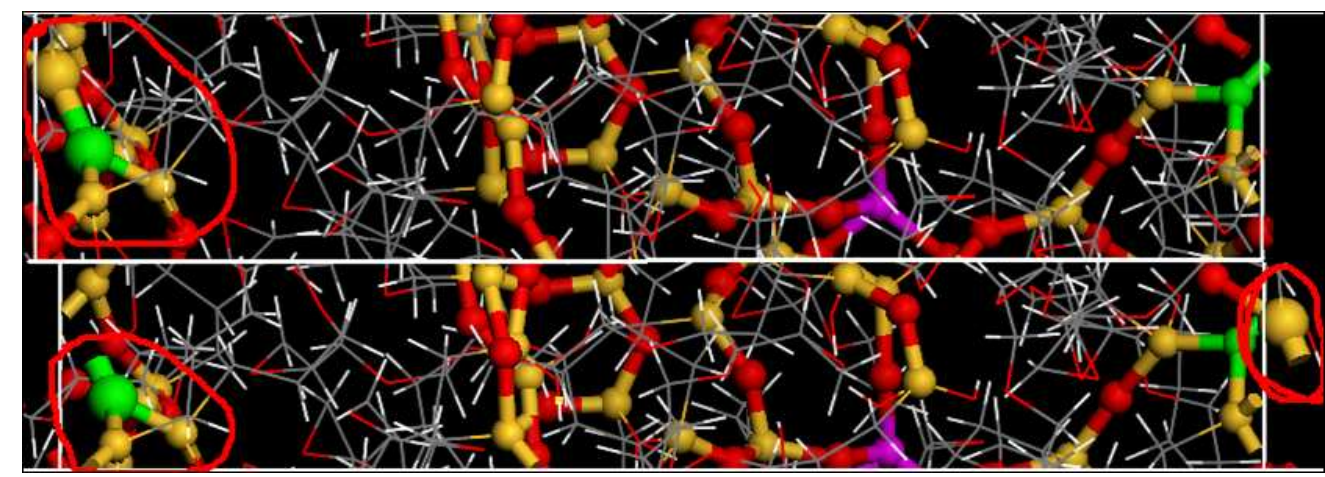

$160 \times 56 \mathrm{~mm}(132 \times 132 \mathrm{DPI})$ 


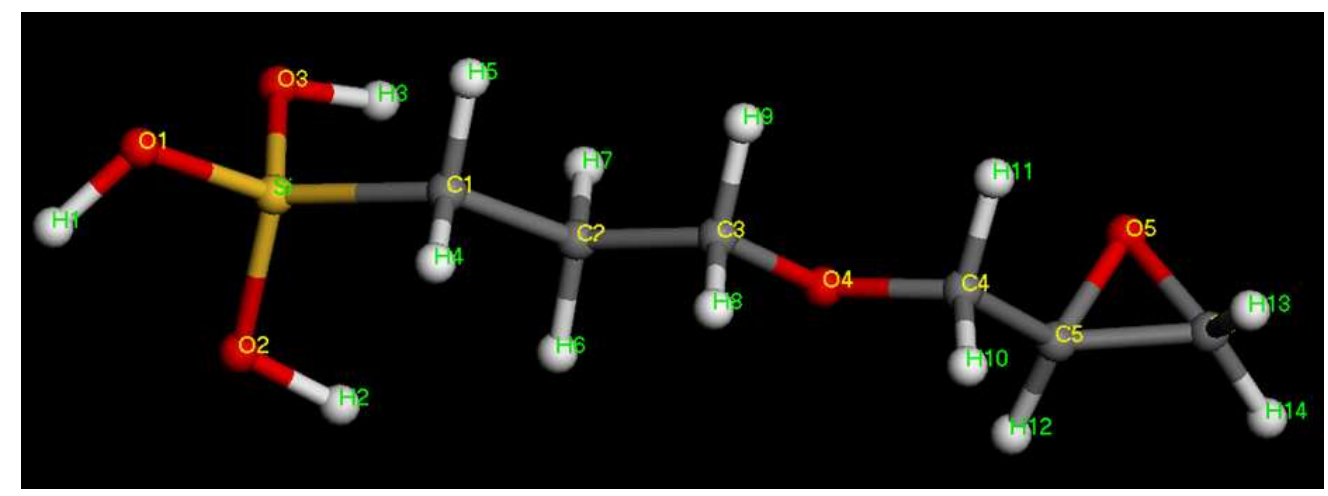

$156 \times 57 m m(150 \times 150$ DPI $)$

2

4

5

6

9

10

11

13

14

15

16

17

18

19

20

21

22

23

24

25

26

27

28

29

30

31

32

33

34

35

36

37

38

39

40

41

42

43

44

45

46

47

48

49

50

51

52

53

54

55

56

57

58

59

60

http://mc.manuscriptcentral.com/tandf/jenmol 


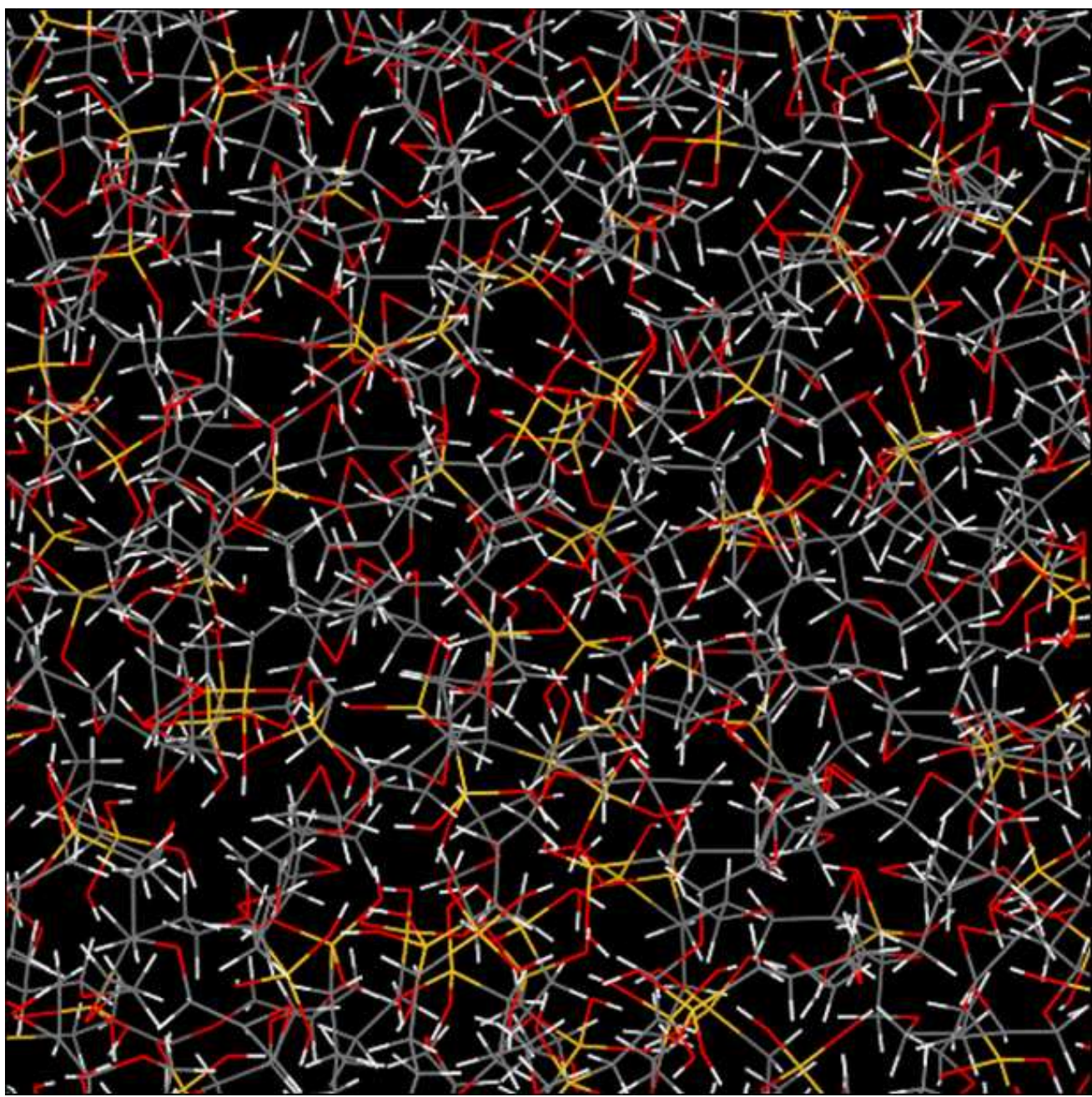

$103 \times 104 \mathrm{~mm}(150 \times 150$ DPI $)$ 


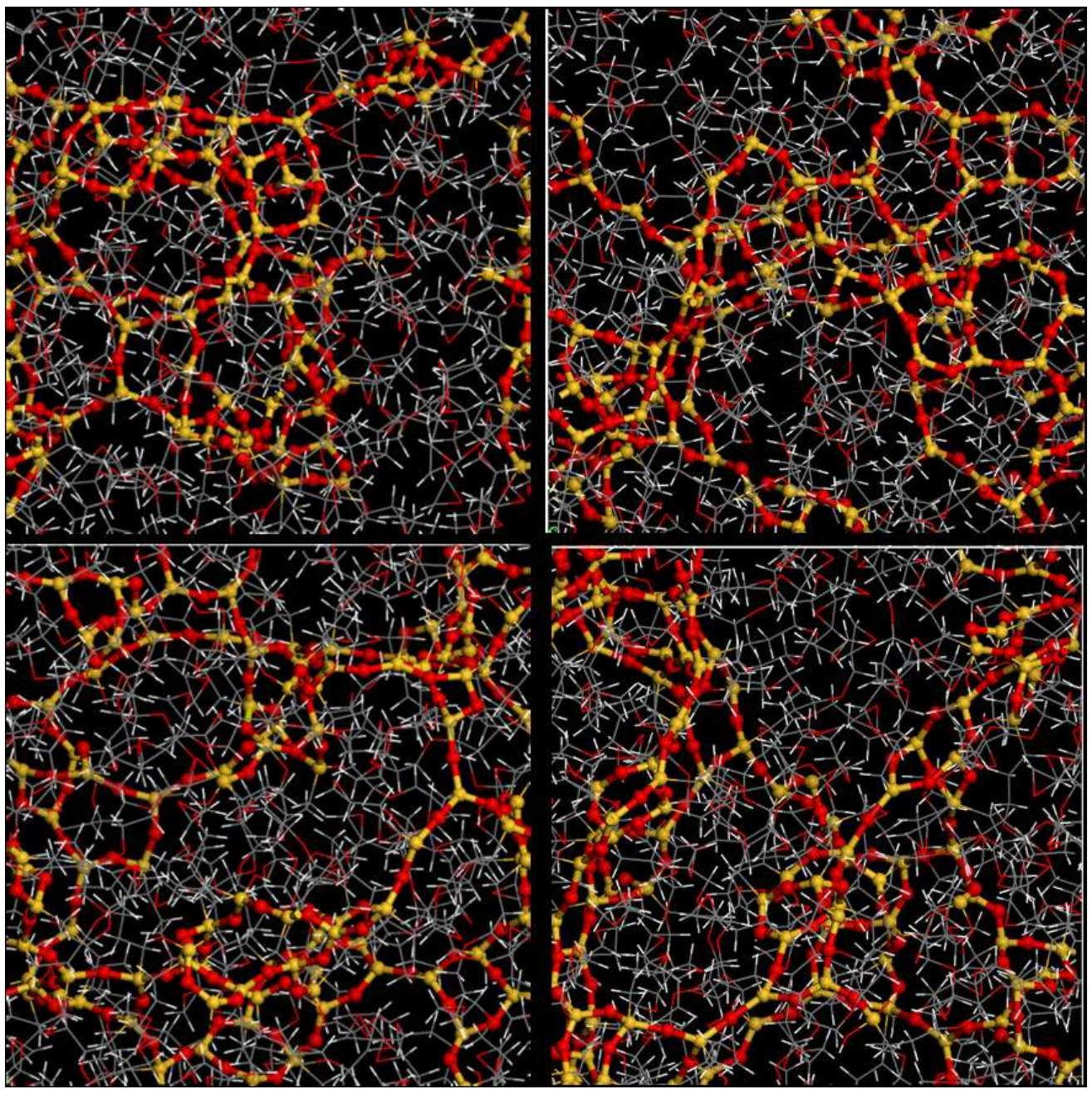

$148 \times 148 \mathrm{~mm}(150 \times 150 \mathrm{DPI})$ 


\section{Page 39 of 39}

1
2
3
4
5
6
7
8
9
10
11
12
13
14
15
16
17
18
19
20
21
22
23
24
25
26
27
28
29
30
31
32
33
34
35
36
37
38
39
40
41
42
43
44
45
46
47
48
49
50
51
52
53
54
55
56
57
58
60

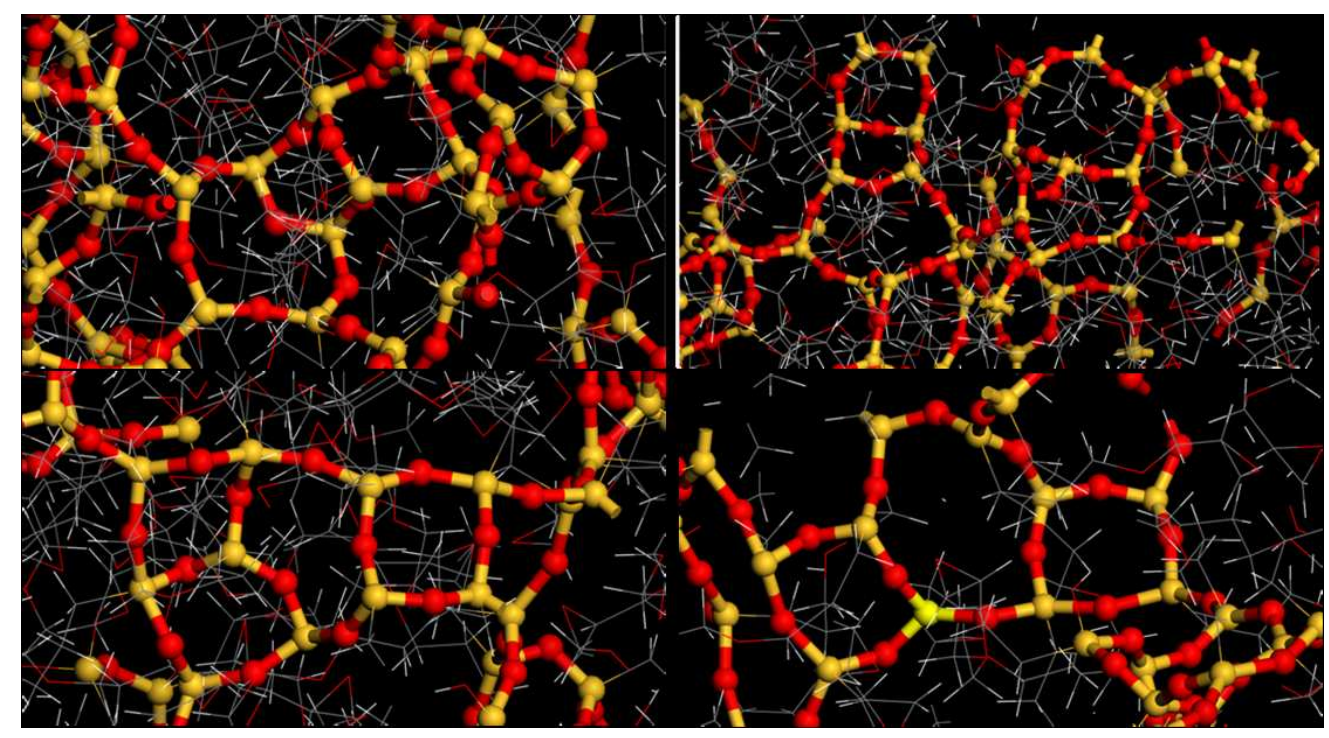

$188 \times 103 \mathrm{~mm}(150 \times 150 \mathrm{DPI})$ 